Portland State University

PDXScholar

Civil and Environmental Engineering Faculty

Publications and Presentations

Civil and Environmental Engineering

$1-1-1990$

\title{
Residual Circulation in Shallow Estuaries 1. Highly Stratified, Narrow Estuaries
}

\author{
David A. Jay \\ Portland State University \\ J. Dungan Smith \\ University of Washington
}

Follow this and additional works at: https://pdxscholar.library.pdx.edu/cengin_fac

Part of the Civil and Environmental Engineering Commons

Let us know how access to this document benefits you.

\section{Citation Details}

Jay, D. A., and J. D. Smith (1990), Residual Circulation in Shallow Estuaries 1. Highly Stratified, Narrow Estuaries, Journal of Geophysical Research, 95 (C1), 711 - 731.

This Article is brought to you for free and open access. It has been accepted for inclusion in Civil and Environmental Engineering Faculty Publications and Presentations by an authorized administrator of PDXScholar. Please contact us if we can make this document more accessible: pdxscholar@pdx.edu. 


\title{
Residual Circulation in Shallow Estuaries 1. Highly Stratified, Narrow Estuaries
}

\author{
David A. Jay and J. Dungan Smith
}

Geophysics Program, University of Washington, Seattle

\begin{abstract}
Long-wave theory and simple turbulence closures have been used to show that three distinct types of circulation (highly stratified, weakly stratified, and partially mixed) arise in narrow, shallow estuaries from the finite amplitude of the tide and the interaction of stratification with vertical mixing. Each type has a different dominant process causing the vertical exchange of salt and fresh water on the flood, and each gives rise to a characteristic residual circulation. The tidal circulation in highly stratified shallow estuaries, described herein, is the result of a finite amplitude internal motion driven by the barotropic tide; shear instabilities at the interface are the major vertical exchange mechanism. The residual circulation is caused primarily by ebb-flood asymmetry in interface position and thickness. A model based on our theoretical analysis shows that the interface thickness on flood is much less than the depth of flow up to a critical tidal amplitude at which the two-layer flow is destroyed, and correctly predicts the tidal amplitude of the neap-spring transition in the Columbia River Estuary. Tidal frequency internal wave motion is not found in weakly stratified and partially mixed estuaries. Because of the reduced tidal shear, shear instabilities are weak or absent, and the residual circulation assumes a very different character, as described in part 2.
\end{abstract}

\section{Introduction: Definition of the Residual Flow Problem}

The residual circulation is the time average of the velocity and density fields over one or a few tidal cycles. This definition encompasses both the effects of forcing mechanisms with time scales long relative to the tidal cycle (e.g., river flow fluctuations) and of nonlinearities in the tidal circulation itself (e.g., the finite amplitude of the barotropic and internal tides). The residual circulation changes, therefore, over the tidal month as the tidal amplitude varies and in response to changes in river flow, storms, and other low-frequency processes. Residual flows are important for the role they play in a broad range of estuarine phenomena including dispersion of salt, turbidity maximum generation, phytoplankton dispersion, and pollutant and suspended sediment transport. Their understanding is also an exciting fluid mechanical challenge because of the multiple, nonlinear driving mechanisms present in most estuaries.

Residual circulation in shallow, stratified estuaries arises primarily from the interaction of the density field, river flow, and tidal currents. Both barotropic and baroclinic mechanisms are important, but to varying degrees in different systems. While systems exist in which the residual circulation is dominated by only one of the above factors - for example, river flow overwhelms all other processes in the Amazon River - the mean circulation in most shallow estuaries is produced by a combination of several factors. If the tidal forcing is strong, the interaction of the density field, river flow, and tidal currents usually predominates, with atmospheric forcing playing a secondary role. This is the situation considered herein.

Copyright 1990 by the American Geophysical Union.
Most previous theoretical analyses of residual flow have concentrated on only one aspect of the residual circulation rather than on the residual circulation as a whole. Neglect of joint effects of tidal flow and the density field has led to an unfortunate separation of the barotropic and baroclinic residual flow generation problems. Either the density has been assumed uniform and residual flow hypothesized to result from barotropic nonlinearities associated with the finite amplitude of the tidal wave, or the mean flow and density fields were assumed to maintain one another more or less directly, with the tidal influence being treated only parametrically. Moreover, no existing theory of estuarine residual circulation incorporates modern insights into mixing processes in stratified flows to distinguish the various types of estuaries. We suggest a different point of view: the residual circulation arises from ebb-flood asymmetry in both transport and mixing. Bottom boundaryinduced turbulence, Kelvin-Helmholtz instabilities, random internal wave interactions, and perhaps other mechanisms all play a role in vertical exchange in stratified estuarine flows [Gardner et al., 1980; Gargett, 1984]. Abraham [1988] has argued that even the most complex numerical models are presently unable to represent the full spectrum of relevant mixing processes. Resolution of these difficulties requires, we believe, primarily new physical insight rather than more sophisticated numerical methods. We have sought, therefore, to represent analytically the dominant features of wave propagation and vertical momentum exchange in two-dimensional (in the along-channel and vertical directions), shallow estuaries.

Our approach is to apply well-established methods of surface and internal long-wave theory, and simple turbulence closures to describe the tidal and residual circulation in shallow estuaries. This extension of wave methodology to the internal circulation is justified both by the observed wavelike nature of the advance and retreat of the salt water mass in highly stratified estuaries [Geyer, 1985, 1988] and by the success of Ianniello $[1977 a, b]$ in using wave methodology to analyze barotropic residual flow in estu- 
aries. The analogy between barotropic and internal tidal phenomenon is examined in detail in section 3.

More specifically, this contribution and that of Jay and Smith [this issue] (hereinafter referred to as part 2) show that the finite amplitude of the barotropic and internal tides and the nonlinear interaction of stratification with vertical mixing cause three different modes of circulation to appear in shallow, stratified estuaries. Each of these types has a characteristic vertical exchange mechanism that mixes salt and fresh water on the flood and a distinctive residual flow pattern. The mixing on flood is of particular importance, because model results and observations (section 2) show that the flood flow patterns are quite distinctive, while ebb flow patterns for the three types of estuaries show a greater degree of commonality. The flood is therefore decisive for the evolution of the residual flow.

Two of the three types of estuaries (weakly stratified and partially mixed) can be described as the sum of a tidal and residual barotropic mode interacting weakly with a baroclinic mode forced by a steady horizontal salinity gradient (part 2). Tidal frequency baroclinic flow is absent, and the ebb-flood asymmetry is primarily associated with the mean density gradient. The barotropic mode in these estuaries is essentially that described by Ianniello [1977a, b]. The baroclinic component is similar to that of "central regime theory" [Hansen and Rattray, 1965], which has been the accepted model of baroclinic estuarine circulation for more than 2 decades. The weakly stratified and partially mixed regimes are distinguished by the presence in the former of vertical turbulent mixing in the interior as the dominant process mixing salt and fresh water on flood. Stratification is too strong relative to shear in partially mixed estuaries for bottom boundary-induced turbulence to be present, except near the bed. The exchange of salt and fresh water in the interior is, therefore, much weaker than in weakly stratified systems. We hypothesize that random internal wave interaction is the primary vertical exchange mechanism in the interior of partially mixed estuaries, but other weak mechanisms may also be active.

The third circulation type (highly stratified or salt wedge) has, in contrast, a strong baroclinic tidal component [Geyer, 1985, 1988]. The advance and retreat of a salt wedge must therefore be treated as a baroclinic tidal wave forced by the barotropic tide. The residual flow arises largely from the ebb-flood asymmetry in interface position and thickness. The dominant vertical exchange mechanism is Kelvin-Helmholtz (shear) instabilities on the interface. While bottom boundary-induced turbulence is present in the lower layer, it cannot be the agent responsible for the mixing of salt and fresh water, because of the strong stratification in the interfacial layer. Conversely, shear instabilities cannot be the primary vertical exchange mechanism in weakly stratified or partially mixed estuaries because there is no strong, tidal-frequency internal circulation to generate strong shear in the interior, where the mixing of salt and fresh water occurs.

The present study applies to tidal rivers, drowned river valley estuaries, and other narrow, channel-like systems. Broad tidal flats may be present, so long as most of the mass and salt exchange is confined to the channels. The requirement that the circulation be essentially two dimensional in the along-channel and vertical directions excludes those systems that are extensive in two horizontal directions, in which the salt balance may be maintained by chaotic dispersion mechanisms associated with topographic generation of residual vorticity [Zimmerman, 1980, 1986]. Jay [1989] has shown that these chaotic dispersion mechanisms cannot operate in narrow channels (as defined in section 3 ) in which the cross-channel circulation is weak. Furthermore, there are very few rivers in the world with sufficient river flow to sustain a broad, saltwedgelike flow that do not have sufficient sediment load to cause formation of a delta made up of a series of narrow channels. The Amazon is perhaps the only river system with a unobstructed mouth broad enough that chaotic dispersion processes are likely to be consistently important but the river flow in the Amazon is so large that salinity intrusion is absent. Thus the two dimensional model employed in the present study is probably a good approximation for most shallow, highly stratified, salt wedge systems.

Our theoretical approach emerged from an observational study of residual circulation in the Columbia River Estuary, which, in turn, was motivated by a desire to understand the tidal monthly changes in the density field and strength of the upstream bottom flow. These do not vary smoothly during the tidal month but exhibit sharp transitions at certain critical tidal ranges (Figure 1). A satisfactory understanding of these transitions requires identification of the basic driving mechanism thereof. The obvious possibilities are instability of the flow over topographic highs as is commonly observed in fjords [Farmer and Freeland, 1983], and a global breakdown of stratification coupled with a change to a different, dominant vertical exchange mechanism.

Tidal monthly changes in the density field and circulation patterns are also found in numerous other river estuaries, for instance, some of the tributaries of Chesapeake Bay and the macrotidal estuaries of England and France [Haas, 1976; Allen et al., 1980]. The number of systems in which such tidal monthly transitions occur and the diversity of their morphology argue against the idea that these changes are related to or controlled by specific topographic features. The occurrence of weak stratification throughout the Columbia River Estuary immediately after the spring-neap transition (section 2 and Jay [1987]) also suggests a global breakdown of stratification in the system, rather than a local breakdown at a single point. If this mixing were a purely local phenomenon caused by a bar or constriction, then the horizontal density gradient resulting from the mixing would cause restratification upstream of the point of breakdown on flood. This would occur both because the flow would become less energetic away from the bar or constriction, and because the ratio of river flow to tidal exchange increases in the upriver direction. Such restratification is not, in fact, observed. Moreover, an a posteriori argument can be made. Were topography the primary feature governing changes in the character of the baroclinic circulation, then the uniform depth and width models used herein and in part 2 would probably not be successful in predicting the tidal range at which tidal monthly transitions occur. Finally, scaling arguments (section 3) suggest that topographic effects are 


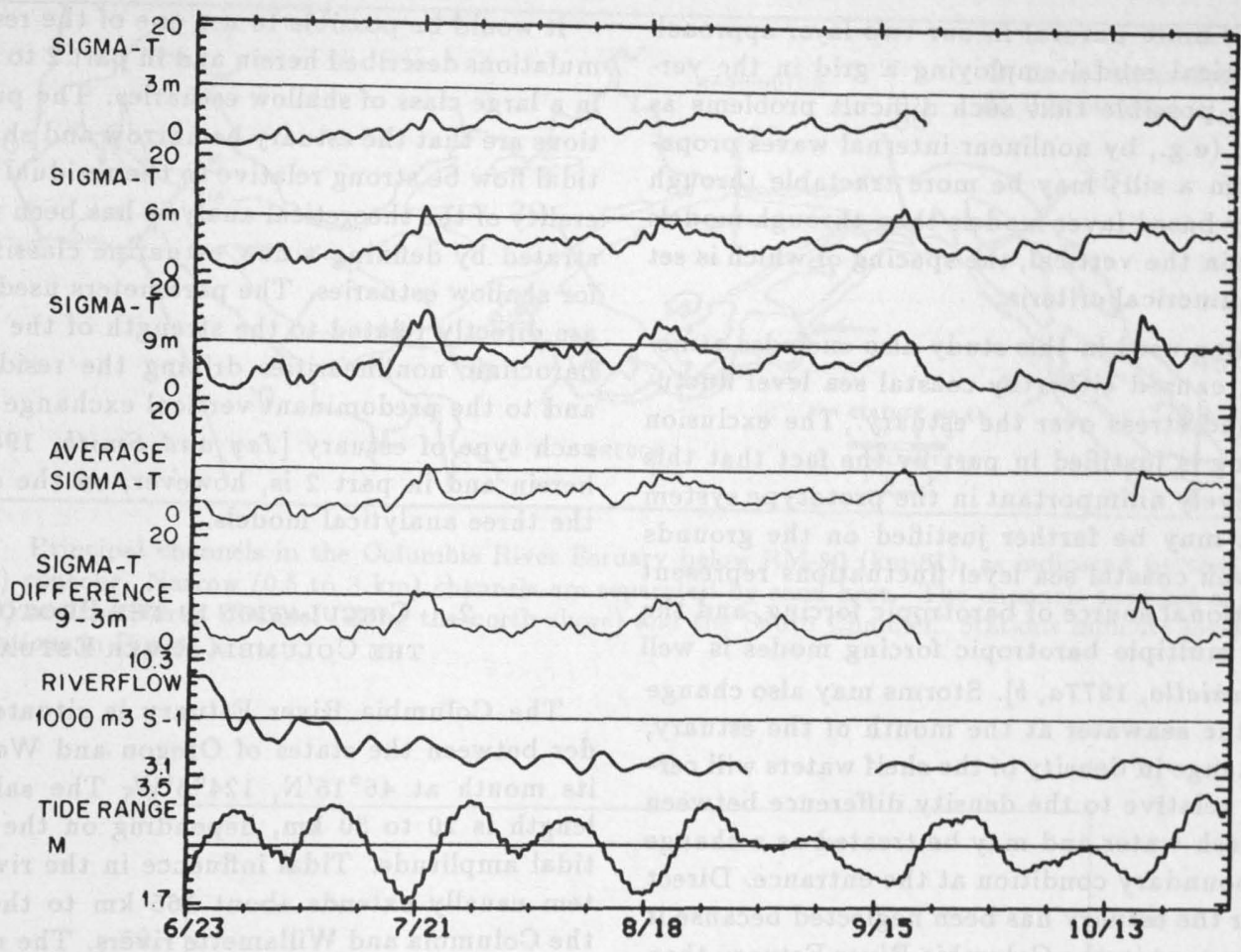

Fig. 1. Time series for the summer and fall seasons of (top to bottom) low-passed (tidal signal removed) density $\left(\sigma_{t}\right)$ in midestuary at 3,6 , and $9 \mathrm{~m}$, vertical average of low-passed density at 3,6 , and $9 \mathrm{~m}$, low-passed density difference $(9-3 \mathrm{~m})$; river flow, and tidal range. The three density records are from a station at RM(river mile)-13 $(\mathrm{km}-21)$ in the South Channel. Tidal range is calculated from harmonic analyses of observations collected by the National Ocean Service at RM-18 (km-29) and river flow from records at Bonneville Dam (RM-146; km-235) and the mouth of the Willamette River (RM-101; km-164). Station locations are shown in Figure 2. The density increases as the river flow decreases early in the record, and the stratification is governed primarily by the tidal range. Sharp increases and decreases in density (time scale of days) indicate tidal monthly transitions. The vertical averaged density is greater during highly stratified, neap tide periods because reduced bottom stress and increased baroclinic forcing allow greater salinity intrusion length, despite the small tidal range.

not dominant in shallow, stratified estuaries. Thus we believe that instability of the flow throughout the system and consequent changes in the vertical momentum and salt exchange mechanisms as a function of river flow, tidal range, and baroclinic forcing are the critical factor in causing the observed tidal monthly transitions. This distinguishes shallow estuaries from fjords and other deep estuaries, where mixing is often controlled by sills.

Consistent with this assumption, channel width and depth were held constant in the calculations discussed herein. This allows the interaction of the density field, river flow, and tides to be the dominant feature in the resulting models and facilitates application of long-wave methodology. The conceptual richness and power of wave theory allows these simple models to provide major insights into estuarine circulation, including a new estuarine classification system [Jay and Smith, 1988]. The resulting models are also believed to be the simplest possible representation of estuarine residual circulation that allows investigation of the tidal monthly transitions. This approach by no means excludes the possibility, even probability, that topographic features exert a substantial but not dominant influence on tidal monthly transitions and the baroclinic circulation in many shallow systems. One of the advantages of the use of long-wave theory, however, is the extensive body of available methods. The results derived here can probably be extended to include at least modest topographic features using methods similar to those developed for the barotropic problem by Ianniello $[1979,1981]$. Strong bathymetric changes (e.g., fjord sills) would require segmentation of a two-layer model. Analogous barotropic methods are also available here [e.g., Dronkers, 1964].

The two-layer model used here also possesses, despite the simplified topography, a definite advantage over more conventional models (those using finite element or finite differences in the vertical) with regard to calculating internal mixing driven by Kelvin-Helmholtz instabilities, the dominant vertical exchange mechanism in highly stratified estuaries. Turbulence closures based on parameterization of the turbulent kinetic energy equation still do not, at least as used in numerical models, give entirely satisfactory results in reproducing internal mixing in free shear layers [Abraham, 1988]. We contend that it is probably more accurate and certainly far simpler to parameterize internal mixing directly from the interfacial properties that drive the Kelvin-Helmholtz instabilities and thus govern this mixing than from any presently available parameterization of the turbulent kinetic energy equation. This is done in section 3 by using a bulk Richardson number closure [Geyer, 1985; Geyer and Smith, 1987] that makes use of the observed linearity of interfacial density and velocity profiles and incorporates both mean and fluctuating shear. Moreover, use of a bulk Richardson number 
calculation is far more natural in our two-layer approach than in a numerical model employing a grid in the vertical. It is even possible that such difficult problems as nonlocal mixing (e.g., by nonlinear internal waves propagating away from a sill) may be more tractable through use of physically based layer models than through models with fixed grids in the vertical, the spacing of which is set by essentially numerical criteria.

The formulation used in this study also excludes atmospheric forcing, caused either by coastal sea level fluctuations or by wind stress over the estuary. The exclusion of coastal forcing is justified in part by the fact that this process is relatively unimportant in the prototype system [Jay, 1987]. It may be further justified on the grounds that storm-driven coastal sea level fluctuations represent simply an additional source of barotropic forcing, and the joint action of multiple barotropic forcing modes is well understood [Ianniello, $1977 a, b]$. Storms may also change the density of the seawater at the mouth of the estuary, but any such change in density of the shelf waters will certainly be small relative to the density difference between seawater and fresh water and may be treated as a change in the density boundary condition at the entrance. Direct wind stress over the estuary has been neglected because it is even less important in the Columbia River Estuary than are coastal sea level fluctuations. Wind stress may, however, be considered to drive a linearly separable mode and could be added to the analytical solutions derived herein, except in systems where the strength of the atmospheric forcing approaches that of the tidal forcing. In the latter case, the wind stress becomes an inseparable part of the lowest order flow through its enhancement of the mean shear and hence of the vertical mixing.

The major difficulty in an analytical investigation of the residual flow problem is the form of the density field. If nothing is assumed about the form of the density field and the nature of the mixing, then the interaction of stratification and mixing is analytically intractable. In fact, however, observations described in section 2 show that the density field in the Columbia River Estuary alternates in a regular way between highly stratified (around the neap tide) and weakly stratified (most of the rest of the tidal month; Figure 1). The partially mixed transitions between the two states last only a few days (perhaps one to two flushing times [Neal, 1972]). Each of these conditions has, moreover, a dominant vertical mixing process. Simple approximations to the density field and mixing processes in each state may then be incorporated into analytical models. The fall, low-flow period constitutes an ideal test of the theoretical analysis, because neap-spring transitions in stratification occur with regularity and because disruption of the tidal monthly flow cycle by river flow and storm events is at a minimum. The model for the highly stratified state is used herein to predict tidal and residual velocity and density fields and the tidal amplitude of the neap-spring transition. The model for the weakly stratified state (part 2) is then used to predict the property distributions for spring tides and the tidal amplitude of the spring-neap transition. The model for the partially mixed state shows that the transient, partially mixed condition is unstable in the Columbia River Estuary because the river flow and tidal flow are too strong (part 2).
It would be possible to use one of the residual flow formulations described herein and in part 2 to represent flows in a large class of shallow estuaries. The principle restrictions are that the estuary be narrow and shallow, and that tidal flow be strong relative to the residual flow. The generality of the theoretical analysis has been further demonstrated by defining a new estuarine classification system for shallow estuaries. The parameters used in this system are directly related to the strength of the barotropic and baroclinic nonlinearities driving the residual circulation and to the predominant vertical exchange mechanisms in each type of estuary [Jay and Smith, 1988]. The focus herein and in part 2 is, however, on the development of the three analytical models.

\section{Circulation in the Prototype the Columbia River Estuary}

The Columbia River Estuary is situated on the border between the states of Oregon and Washington, with its mouth at $46^{\circ} 15^{\prime} \mathrm{N}, 124^{\circ} 5^{\prime} \mathrm{W}$. The salinity intrusion length is 20 to $50 \mathrm{~km}$, depending on the river flow and tidal amplitude. Tidal influerce in the river-estuary system usually extends about $165 \mathrm{~km}$ to the confluence of the Columbia and Willamette rivers. The most prominent features of the circulation in the Columbia River Estuary are the high energy level, strong salinity gradients and short flushing time provided by the strong tidal currents and large river flow. The lower reach (about $75 \mathrm{~km}$ ) of the river and estuary consists of narrow $(0.5$ to $3 \mathrm{~km})$ channels separated by islands and broad shoals. Much of the part of the system in which salinity intrusion occurs has two major channels (Figure 2), known as the North and South channels. They divide at about RM(river mile)-7 (km-11) and rejoin at RM-25 (km-40). (The river mile designation of longitudinal position is maintained herein because it appears on navigation charts and has been used in many previous studies.) Throughout the system, flow control structures divert most of the river flow into the main navigation channel, which below RM-25 is the South Channel. Because the tidal flats drain predominantly into the North Channel, much of the tidal exchange between RM-7 and RM-25 takes place in the North Channel. Fine sands are the dominant sediment type in all channels and over the midestuary sand flats. Bed forms varying in height from 0.3 to $2 \mathrm{~m}$ or more are found in the major channels [Sherwood et al., 1989]. Channel cross section (Figure 3 ) is relatively uniform seaward of RM-20 (km-32) except for a wide area in the vicinity of RM-15 ( km-24). It is, therefore, a reasonable first approximation to model tidal processes in the part of the Columbia River Estuary system in which salinity intrusion occurs using a channel of uniform depth and width.

\section{Barotropic Processes}

The hydrologic properties of the Columbia River have been reviewed by Paulsen [1949], Orem [1968] and Sherwood et al. [1989]. It is the largest river draining into the Pacific Ocean in western North America, with a drainage basin of $660,480 \mathrm{~km}^{2}$ and an average flow of about 7300 $\mathrm{m}^{3} \mathrm{~s}^{-1}$. The drainage basin consists of coastal and eastern sub-basins each having distinct climatic and hydrologic characteristics. The coastal sub-basin contains only about $8 \%$ of the total surface area, but contributes almost 


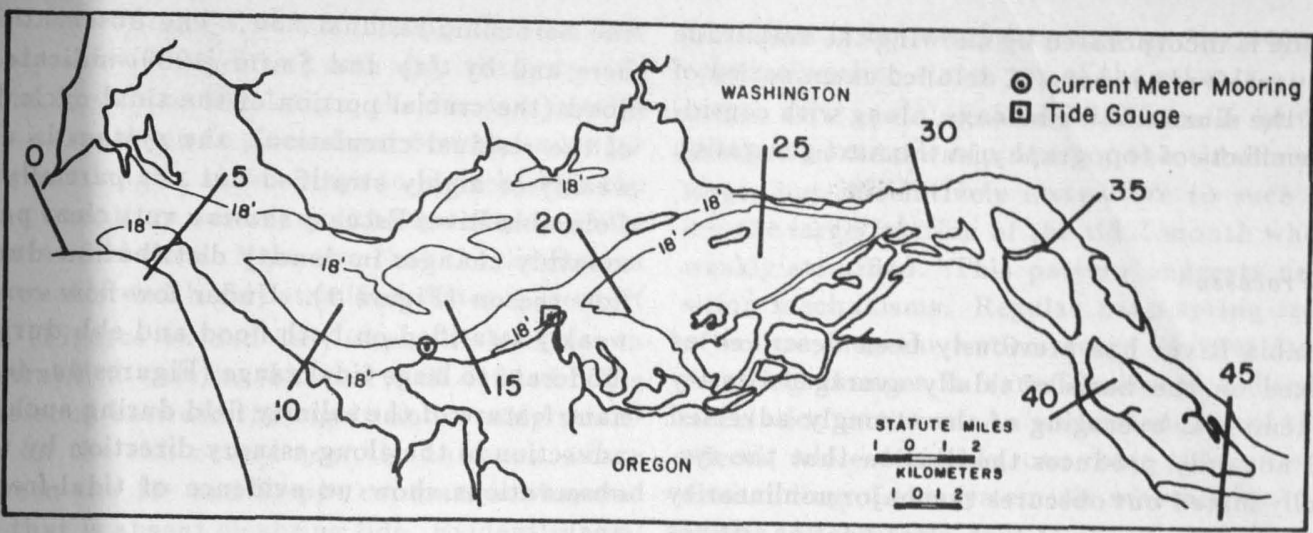

Fig. 2. Principal channels in the Columbia River Estuary below RM-50 (km-81), as indicated by the 18 foot $(5.5 \mathrm{~m})$ contour. Narrow $(0.5$ to $3 \mathrm{~km})$ channels are separated by sand bars. The channels seaward of RM-18 (km-29) are the North Channel (along the north shore) and the South Channel. Stations indicate locations of observations in Figure 1.

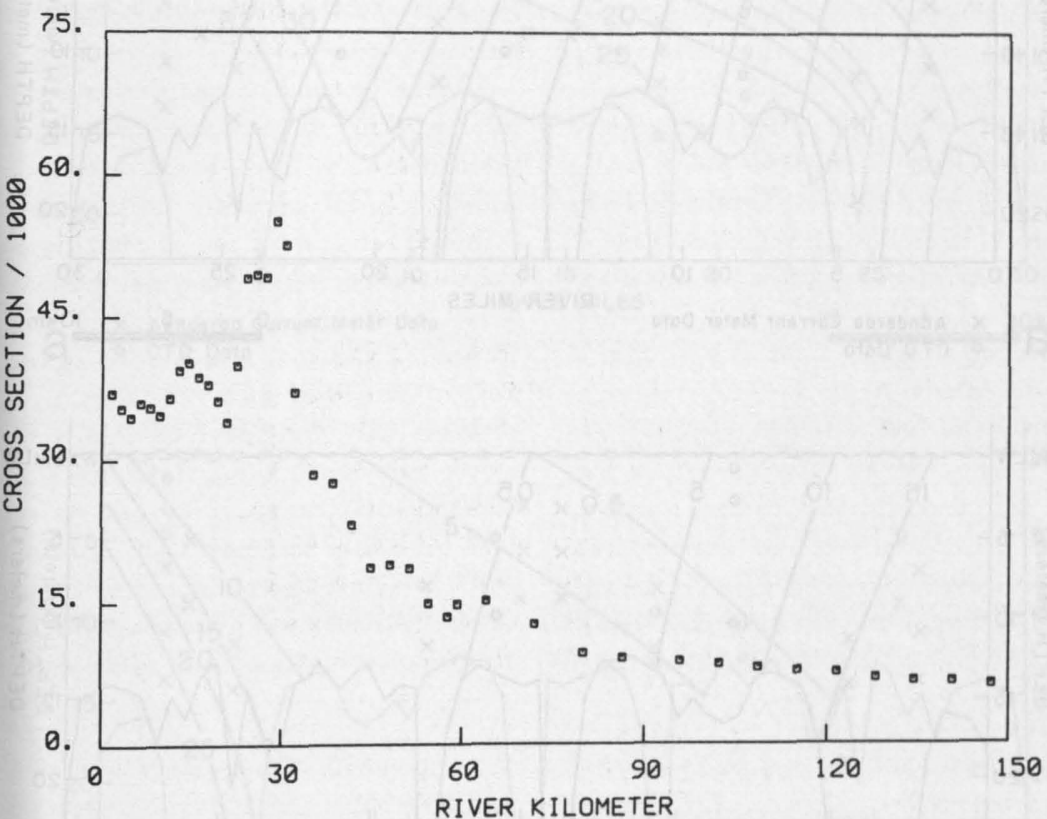

Fig. 3. Cross-sectional area in units of $10^{3} \mathrm{~m}^{2}$ as a function of along-channel distance. Cross-section is nearly constant for the first $25 \mathrm{~km}$, increases briefly, and then decreases exponentially upriver. The exponential decrease is common in tidal river estuaries.

one quarter of the flow. Most of this inflow occurs during the winter. The arid eastern sub-basin has the remaining $92 \%$ of the surface area; most of the runoff occurs during a snowmelt spring freshet in May and June. Because all of the major tributaries are landward of the upstream limits of salinity intrusion, the fresh water inflow can for analytical purposes be assumed to enter at the head of the estuary. Extensive irrigation and flow regulation by dams presently limit the annual range of monthly mean river flow for the entire river basin to a factor of about 3 , from 3000 to $10,000 \mathrm{~m}^{3} \mathrm{~s}^{-1}$. Daily river flow, however, still varies seasonally by about a factor of 5 , from about 3000 to $15,000 \mathrm{~m}^{3} \mathrm{~s}^{-1}$. August to November is the period of both minimum river flow and river flow variability, and storms are uncommon before November. The late summer and fall months are therefore ideal for the study of the interaction between tidal forcing, river flow, and the density field.

The tide at the mouth of the Columbia River Estuary is of the mixed type, with the semidiurnal wave predominating. Tidal properties of the system have been determined by harmonic analyses of current meter and surface elevation records [ Jay, 1984] and through the use of a onedimensional harmonic tidal model [ Jay et al., 1989]. The monthly minimum tidal amplitude is usually between 0.8 and $1 \mathrm{~m}$; the monthly maximum is between 1.5 and $2 \mathrm{~m}$. Almost $90 \%$ of the tidal energy dissipated in the system comes from the principal lunar semidiurnal component $M_{2}$. For this reason, the theoretical models constructed herein and in part 2 treat the residual flow arising from the interaction of a single, semidiurnal constituent with the density field and river flow. Monthly variability in 
tidal amplitude is incorporated by allowing the amplitude of the semidiurnal wave to vary. A detailed examination of the effects of the diurnal wave belongs, along with consideration of the effects of topography, in the next generation of analyses.

\section{Baroclinic Processe}

The Columbia River has previously been descri ${ }^{\not}$ ea as partially mixed on the basis of tidally averaged density data. This temporal averaging of the strongly advected density field not only produces the illusion that the system is partially mixed but obscures the major nonlinearity that at least during periods of high stratification, drives the baroclinic residual flow. The observations discussed here and by Jay and Smith [1989] indicate that on the flood (the crucial portion of the tidal cycle for evolution of the residual circulation), the system is usually either weakly or highly stratified but not partially mixed. The Columbia River Estuary shows a very clear pattern of tidal monthly changes in density distribution during the lowflow season (Figure 1). Under low-flow conditions, it is weakly stratified on both flood and ebb during periods of moderate to large tidal range (Figures $4 a-4 c$ ). The dominant feature of the salinity field during such periods is its advection in the along-estuary direction by the tidal flow; observations show no evidence of tidal-frequency buoyancy.
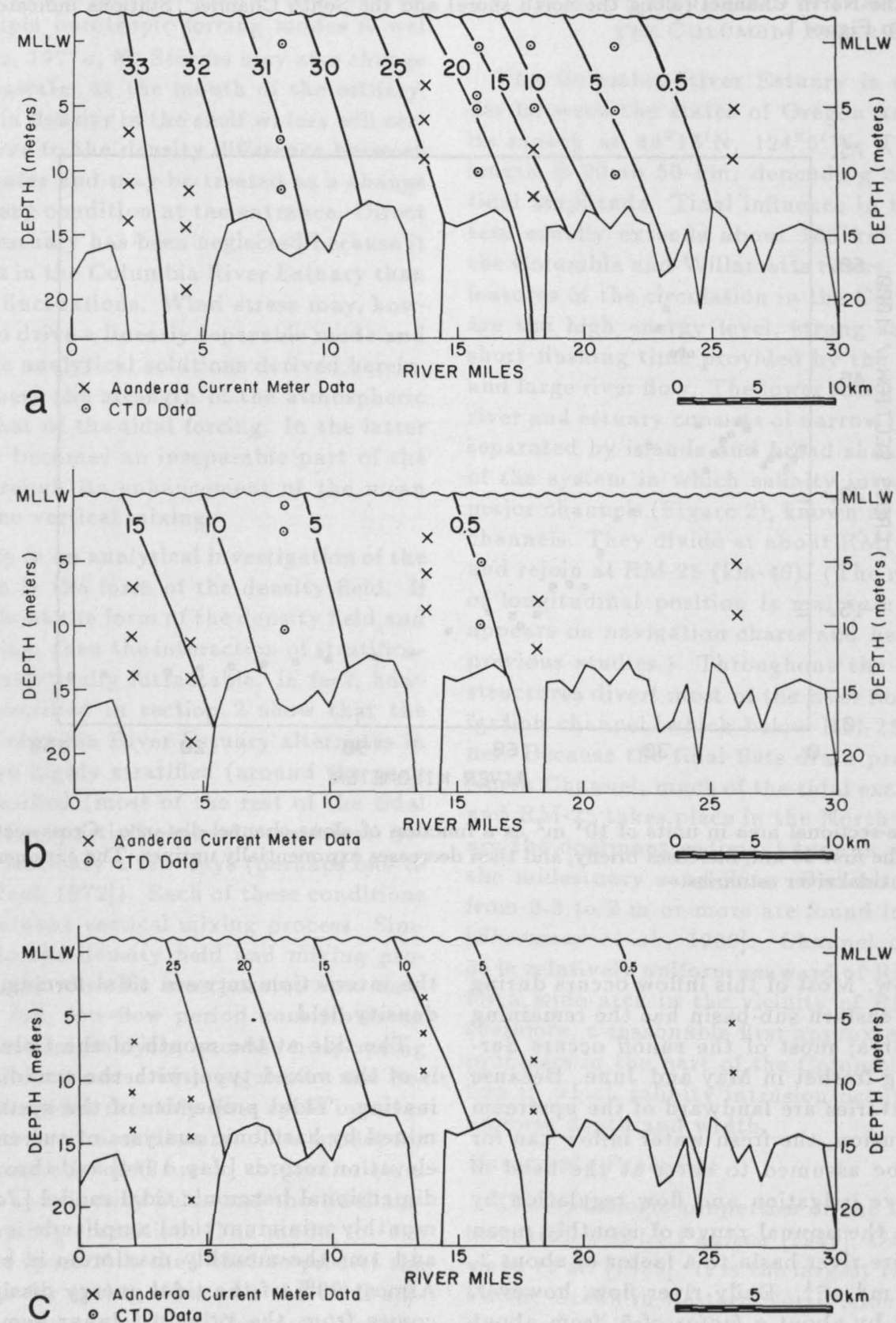

Fig. 4. Salinity sections: $(a)$ end of flood, $(b)$ end of ebb, and $(c)$ tidal cycle mean for the spring tide of October $24-26,1980$. The stratification is weak, and the horizontal density forcing is spatially relatively uniform. The tidal cycle variation in the strength of the horizontal forcing is caused primarily by differential advection. MLLW is mean lower low water. 
Substantial stratification occurs during the low-flow season for only a few days during and after the neap tide (Figures 1 and $5 a-5 c$ ), at which time, the decrease in tidal range causes a transition to a two-layer flow and a longer salinity intrusion length. A few days to a week after the neap, another transition occurs, and the system returns to the weakly stratified state. Note also that the distinction between the weakly and highly stratified states is greatest on the flood (Figures $4 a$ and $5 a$ ), when the interface in the highly stratified case in thin and well defined. The greatest difference between the highly and weakly stratified regimes on the ebb occurs near the entrance, where a front between the 15- and 25-ppt contours is observed on the neap that is absent on spring tide. Evidently, tidal advection is not strong enough on the neap to push this relatively saline water out of the estuary.

The tidal cycle mean stratification is quite sensitive to changes in tidal range during the tidal monthly transitions, but is relatively insensitive to such changes during the large fraction of the tidal month when the flow is weakly stratified. This pattern suggests nonlinear transition mechanisms. Regular neap-spring transitions are, of course, not the entire story. Winter storms and the resulting changes in runoff sometimes disrupt "normal" circulation patterns. An understanding of these transient effects, while desirable, can come only after a better understanding of the systematic seasonal and tidal monthly effects is achieved.
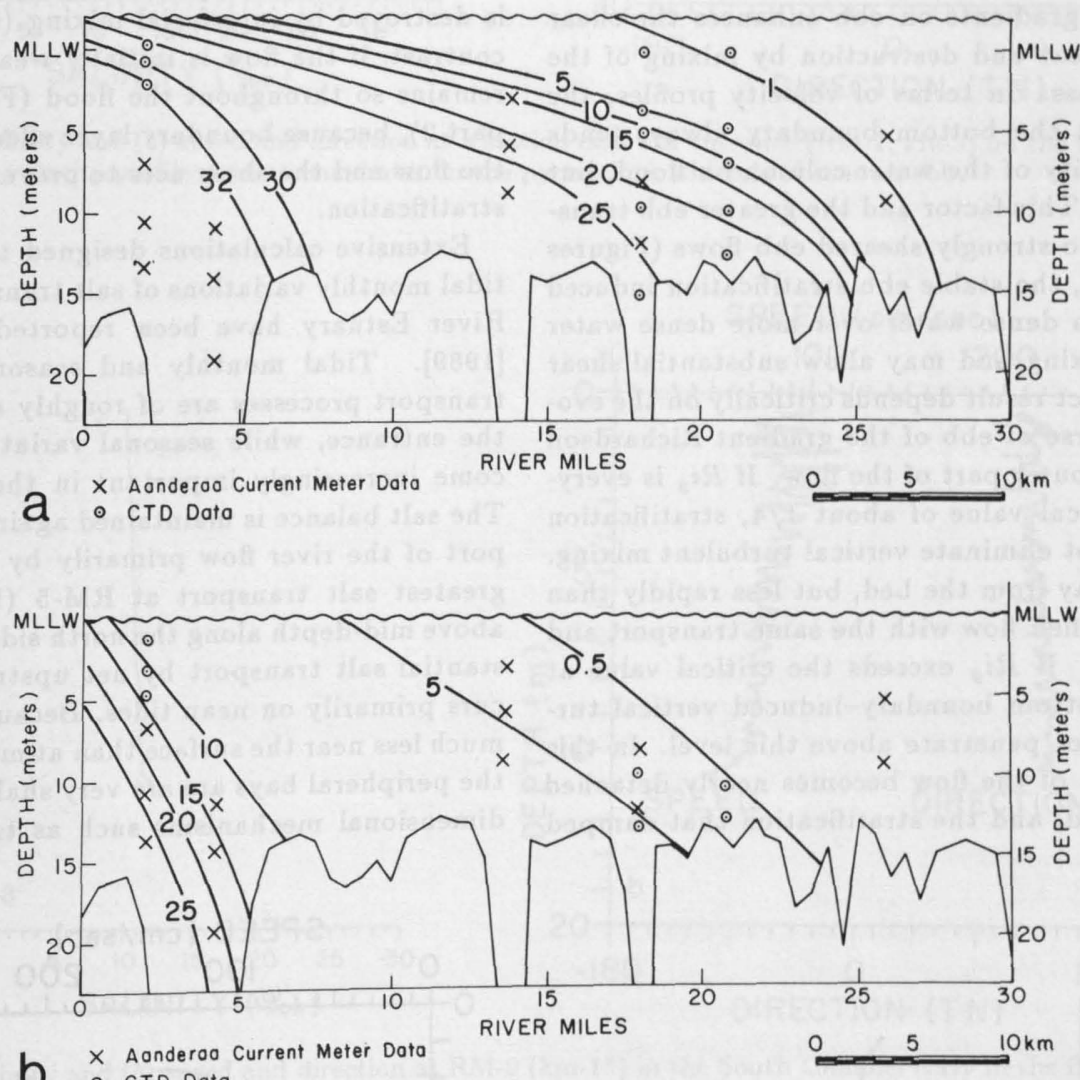

D $\quad \underset{\text { Aanderao }}{\times}$ CTD Data

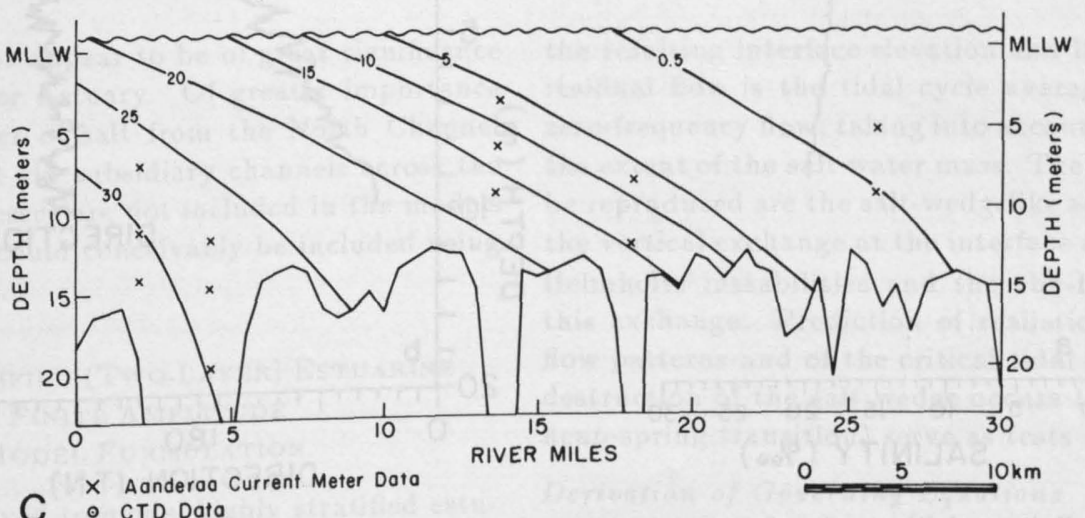

C $\odot$ CTD Data

Fig. 5. Salinity sections: $(a)$ end of flood, $(b)$ end of ebb, and $(c)$ tidal cycle mean for the neap tide of October 16-17, 1980. The flood shows a two-layer advance, with strong horizontal and vertical gradients at the head of the saline water mass. The salt water is both advected and mixed out of the estuary on ebb. 
It will be argued in section 3 and part 2 that estuarine residual circulation arises as the difference between the flood and ebb flows, that is, from the ebb-flood asymmetry. The ebb-flood asymmetry is ultimately caused by the net flux of river water and the resulting horizontal density gradient, which may vary substantially in strength during the tidal cycle but which never changes sign. The difference in ebb and flood transports introduces asymmetries in the bed friction and convective acceleration terms in the equation of motion, even in a neutrally stratified flow. Usually more important in an estuary are the effects of the buoyancy input in establishing a baroclinic pressure gradient and in inhibiting vertical mixing. The baroclinic pressure gradient reinforces the barotropic gradient at depth on flood and makes a salt wedge advance possible. The opposition at depth of the barotropic and baroclinic pressure gradients on ebb enhances the shear and causes the retreat and destruction by mixing of the same salt water mass. In terms of velocity profiles, the shear introduced at the bottom boundary always tends to reduce the stability of the water column on flood, but increases it on ebb. This factor and the greater ebb transport together lead to strongly sheared ebb flows (Figures $6 a$ and $6 b$ ). That is, the stable ebb stratification induced by advection of less dense water over more dense water inhibits vertical mixing and may allow substantial shear to develop. The exact result depends critically on the evolution over the course of ebb of the gradient Richardson number $R i_{g}$ in the outer part of the flow. If $R i_{g}$ is everywhere below a critical value of about $1 / 4$, stratification inhibits but does not eliminate vertical turbulent mixing. Shear decreases away from the bed, but less rapidly than in a neutrally stratified flow with the same transport and over the same bed. If $R i_{g}$ exceeds the critical value at some level, then bottom boundary-induced vertical turbulent mixing cannot penetrate above this level. In this case, the outer part of the flow becomes nearly detached from that at the bed, and the stratification that damped the turbulence is likely to persist through the remainder of the ebb, even when the tidal range is large.

The nature of the flow on flood is strongly influenced by the initial stratification. In highly stratified estuarine flows, the combination of the baroclinic pressure gradient and reduced vertical mixing in the interfacial (pycnocline) region often leads to a distinct velocity maximum near the bottom of the pycnocline [Geyer, 1985]. The flow below is nearly logarithmic, because the lower layer is close to neutrally stratified, and that above is nearly inviscid and without baroclinic forcing. (Figures $7 a$ and $7 b$ ). The enhanced interfacial advection gradually reduces the top-to-bottom density difference at the nose of the wedge, but the two-layer flow is stable throughout the flood as long as the tidal range is sufficiently small. If, however, the barotropic forcing is too strong, then the salt wedge is destroyed by interfacial mixing (sections 3 and 4). In contrast, if the flow is initially weakly stratified, then it remains so throughout the flood (Figures $8 a$ and $8 b$ and part 2), because boundary layer effects extend throughout the flow and the shear acts to prevent the development of stratification.

Extensive calculations designed to assess seasonal and tidal monthly variations of salt transport in the Columbia River Estuary have been reported by Jay and Smith [1989]. Tidal monthly and seasonal variations in salt transport processes are of roughly equal magnitude near the entrance, while seasonal variations in river flow become increasingly important in the upstream direction. The salt balance is maintained against the outward transport of the river flow primarily by tidal advection. The greatest salt transport at RM-5 (km-9) occurs slightly above mid-depth along the north side of the channel. Substantial salt transport by net upstream bottom flow occurs primarily on neap tides. Because salt transports are much less near the surface than at mid-depth, and because the peripheral bays are are very shallow, inherently threedimensional mechanisms such as trapping or storage in
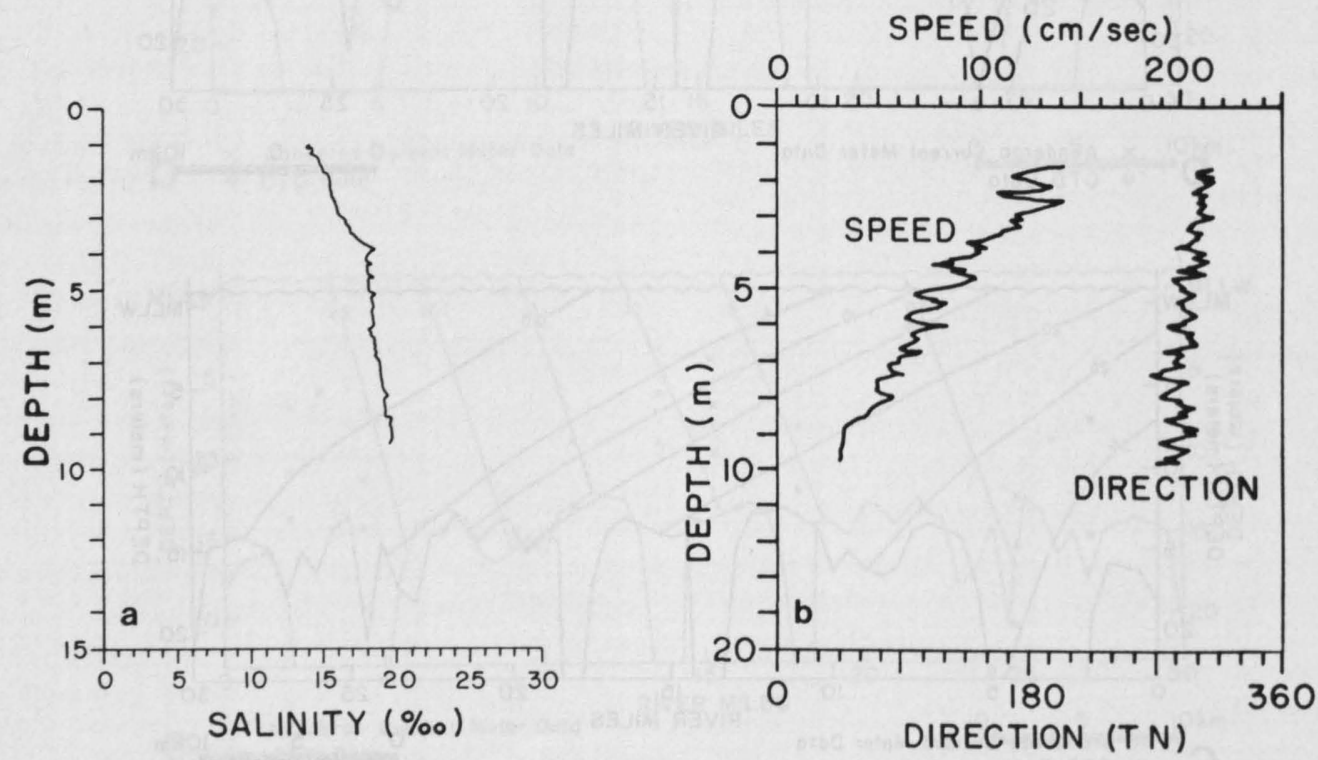

Fig. 6. (a) Salinity and (b) speed and direction at a station in the North Channel at RM-7 (km-12) on the ebb, a few days after the neap. Enhanced stratification and shear in the outer part of the flow is typical; it indicates that this part of the flow is nearly inviscid, despite active mixing throughout the rest of the flow. Much of the noise in the profiles is believed to be caused by ship motion. 

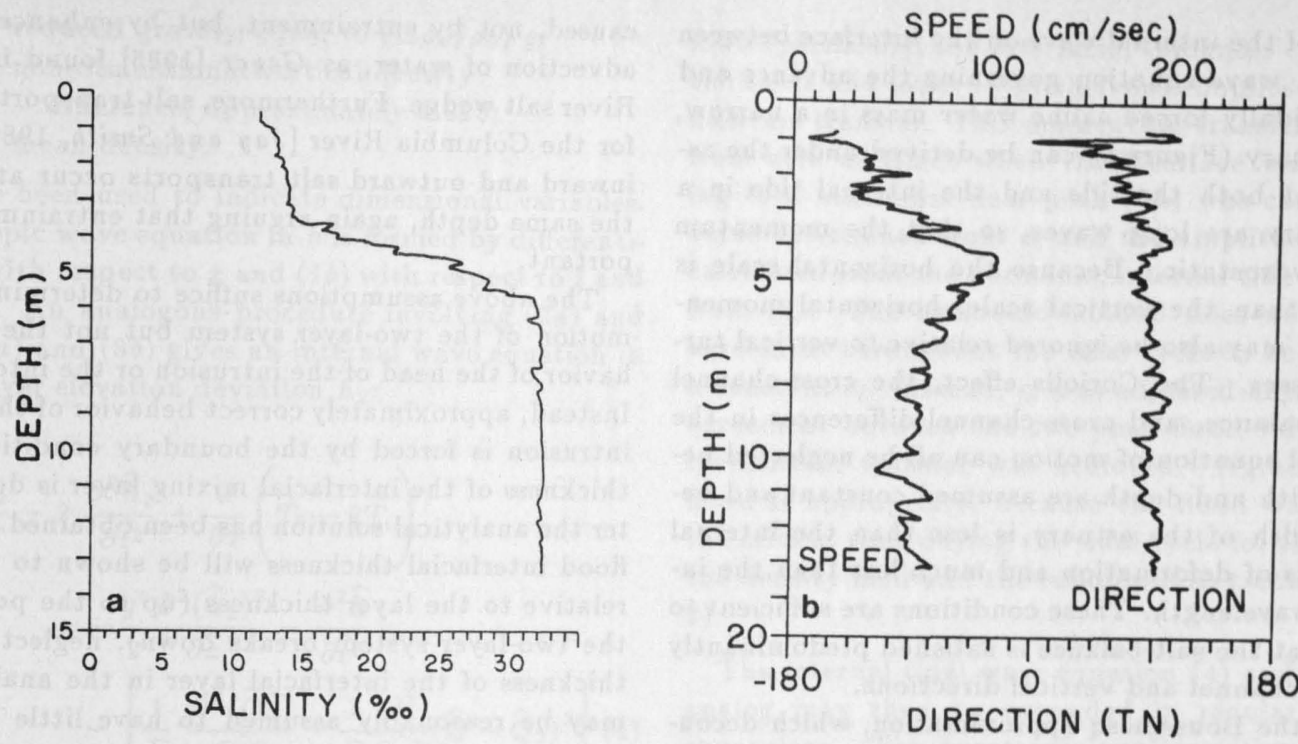

Fig. 7. (a) Salinity and (b) speed and direction at a station near the entrance (RM-2; km-3) on the flood, during the neap. The velocity profile shows a maximum in the pycnocline, which is absent on ebb.
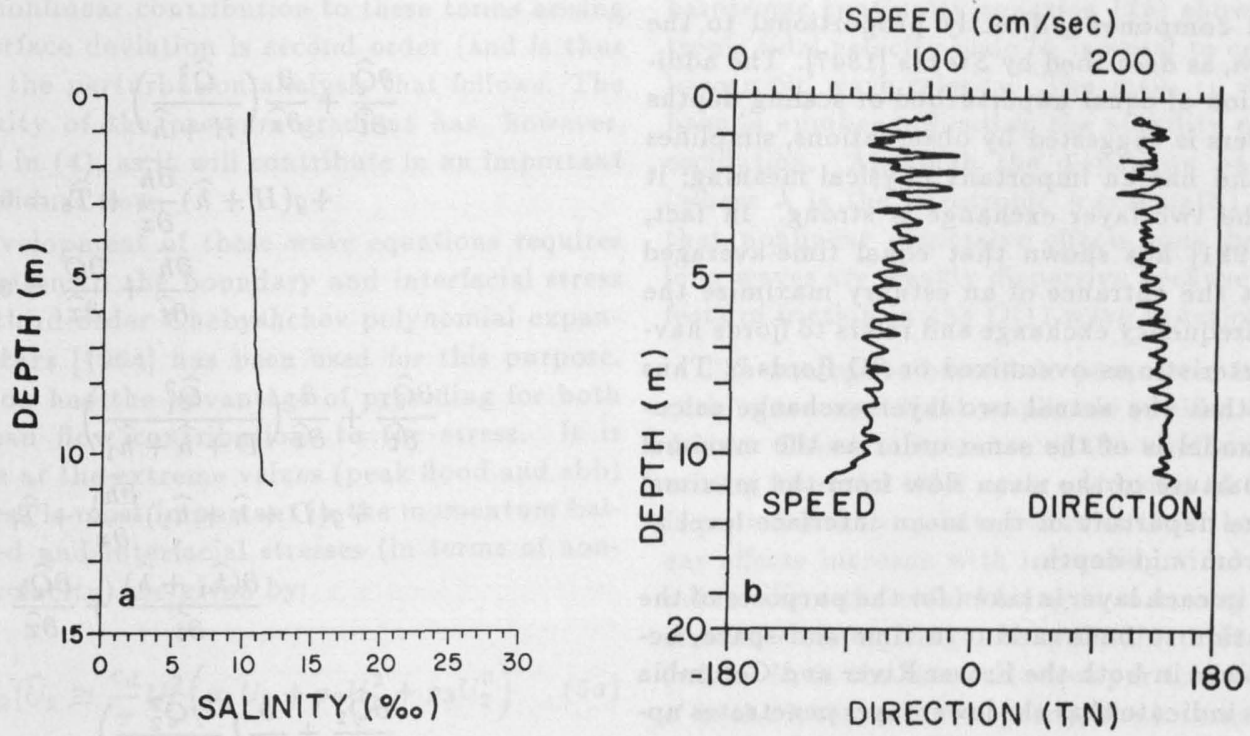

Fig. 8. (a) Salinity and (b) speed and direction at RM-9 (km-15) in the South Channel early in the flood, during a spring tide. The velocity profile is much more uniform, and stratification is minimal.

peripheral bays do not appear to be of great significance in the Columbia River Estuary. Of greater importance is likely to be transfer of salt from the North Channel to the South Channel via subsidiary channels across the tidal flats. Such processes are not included in the models presented herein but could conceivably be included using a network of channels.

\section{Highly Stratified (Two-Layer) Estuarine Flows: Finite Amplitude Wave Model Formulation}

The strategy employed to model highly stratified estuarine flows is to use a perturbation expansion method to find a harmonic solution to a wave equation for two-layer tidal flow, then to calculate the mixing as a function of the resulting interface elevation and layer velocities. The residual flow is the tidal cycle average of the tidal plus zero-frequency flow, taking into account tidal variations in the extent of the salt water mass. The primary features to be reproduced are the salt-wedgelike advance on the flood, the vertical exchange at the interface arising from KelvinHelmholtz instabilities and the ebb-flood asymmetry in this exchange. Prediction of realistic tidal and residual flow patterns and of the critical tidal amplitude at which destruction of the salt wedge occurs through mixing (the neap-spring transition) serve as tests of the model.

\section{Derivation of Governing Equations}

The essence of a salt wedge advance is the combination of barotropic and baroclinic forcing that allows the entire lower layer to advance at a speed approaching the 
phase speed of the internal wave on the interface between the layers. A wave equation governing the advance and retreat of a tidally forced saline water mass in a narrow, two-layer estuary (Figure 9) can be derived under the assumption that both the tide and the internal tide in a shallow estuary are long waves, so that the momentum balance is hydrostatic. Because the horizontal scale is much larger than the vertical scale, horizontal momentum diffusion may also be ignored relative to vertical turbulent processes. The Coriolis effect, the cross-channel momentum balance, and cross-channel differences in the along-channel equation of motion can all be neglected because the width and depth are assumed constant and because the width of the estuary is less than the internal Rossby radius of deformation and much less than the internal tidal wavelength. These conditions are sufficient to guarantee that the salt balance is satisfied predominantly in the along-channel and vertical directions.

We make the Boussinesq approximation, which decouples the barotropic flow from the baroclinic forcing. A rigid lid is not imposed on the baroclinic flow, because the barotropic flow drives the salt wedge advance. The solution for the interface excursion will, as a consequence, consist of the usual incident and reflected free waves plus a third, forced component, directly proportional to the barotropic wave, as described by Stokes [1847]. The additional assumption of equal unperturbed or scaling depths for the two layers is suggested by observations, simplifies the analysis, and has an important physical meaning; it implies that the two layer exchange is strong. In fact, Stigebrandt [1981] has shown that equal time-averaged layer depths at the entrance of an estuary maximize the two-way, zero-frequency exchange and refers to fjords having this characteristic as overmixed or "O-fjords." Thus it is assumed that the actual two layer exchange calculated by the model is of the same order as the maximal exchange. Departure of the mean flow from the maximal level causes the departure of the mean interface level at the entrance from mid-depth.

The density in each layer is taken for the purposes of the analytical solution to be invariant in time and space, because observations in both the Fraser River and Columbia River estuaries indicate that the lower layer penetrates upestuary on flood almost without dilution and with very strong gradients at its head and at the interface (section 2 ; Geyer [1985]). This renders a salt conservation equation unnecessary. Mixing at the interface is allowed, but entrainment is not, becanse the observed weak dilution of the salt water near the head of the salt wedge is probably

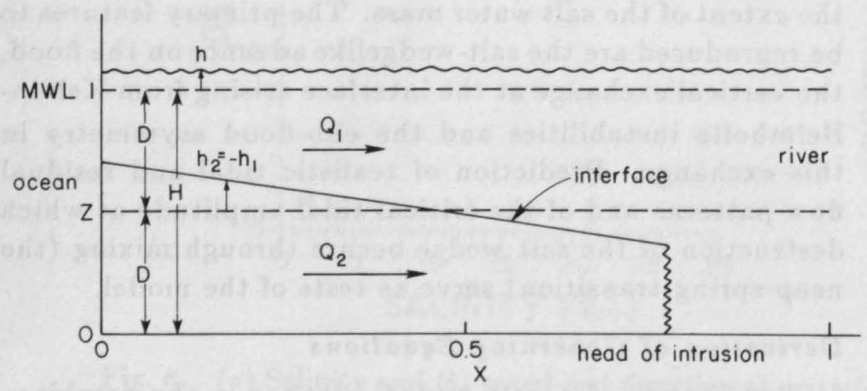

Fig. 9. Schematic of the two-layer system, showing the situation late in the flood. MWL is mean water level. caused, not by entrainment, but by enhanced interfacia advection of water, as Geyer [1985] found in the Fraser River salt wedge. Furthermore, salt transport calculations for the Columbia River [Jay and Smith, 1989] show that inward and outward salt transports occur at very nearly the same depth, again arguing that entrainment is unin portant.

The above assumptions suffice to determine the overall motion of the two-layer system but not the detailed havior of the head of the intrusion or the interfacial laye Instead, approximately correct behavior of the head of th intrusion is forced by the boundary conditions, and t thickness of the interfacial mixing layer is determined ter the analytical solution has been obtained. Because flood interfacial thickness will be shown to remain small relative to the layer thickness (up to the point at which the two-layer system breaks down), neglect of the finite thickness of the interfacial layer in the analytical model may be reasonably assumed to have little effect on the lower layer advance.

The dimensional, vertically integrated momentum and continuity equations for the barotropic flow and in the two layers are (using the subscript " 1 " for the upper layer and " 2 " for the lower layer):

$$
\begin{gathered}
\frac{\partial \widehat{Q}}{\partial \widehat{t}}+\frac{\partial}{\partial \widehat{x}}\left(\frac{\widehat{Q}^{2}}{H+\widehat{h}}\right) \\
+g(H+\widehat{h}) \frac{\partial \widehat{h}}{\partial \widehat{x}}+\widehat{T}_{b}=0 \\
\frac{\partial \widehat{h}}{\partial \widehat{t}}+\frac{\partial \widehat{Q}}{\partial \widehat{x}}=0 \\
\frac{\partial \widehat{Q}_{1}}{\partial \widehat{t}}+\frac{\partial}{\partial \widehat{x}}\left(\frac{\widehat{Q}_{1}^{2}}{D+\widehat{h}+\widehat{h}_{1}}\right) \\
+g\left(D+\widehat{h}+\widehat{h}_{1}\right) \frac{\partial \widehat{h}_{1}}{\partial \widehat{x}}+\widehat{T}_{i}=0 \\
\frac{\partial\left(\widehat{h}_{1}+\widehat{h}_{)}\right.}{\partial \widehat{t}}+\frac{\partial \widehat{Q}_{1}}{\partial \widehat{x}}=0 \\
\frac{\partial \widehat{Q}_{2}}{\partial \widehat{t}}+\frac{\partial}{\partial \widehat{x}}\left(\frac{\widehat{Q}_{2}^{2}}{D+\widehat{h}_{2}}\right) \\
+\left(D+\widehat{h}_{2}\right)\left(g^{\prime} \frac{\partial \widehat{h}_{2}}{\partial \widehat{x}}+g \frac{\partial \widehat{h}_{2}}{\partial \widehat{x}}\right)-\widehat{T}_{i}+\widehat{T}_{b}=0 \\
\frac{\partial \widehat{h}_{2}}{\partial \widehat{t}}+\frac{\partial \widehat{Q}_{2}}{\partial \widehat{x}}=0
\end{gathered}
$$

where

$$
\begin{aligned}
& \widehat{Q} \quad \widehat{Q} \text { barotropic transport; } \\
& \widehat{Q}_{1}, \widehat{Q}_{2} \text { the upper and lower layer transports, } \\
& \text { respectively; } \\
& \widehat{x} \text { horizontal distance; } \\
& \widehat{t} \text { time; } \\
& \widehat{h}_{1}, \widehat{h}_{2} \text { the upper and lower layer depth deviations } \\
& \widehat{h} \text { surface elevation deviation; } \\
& \widehat{T}_{i} \text { interfacial momentum exchange divided by } \\
& \text { the density; } \\
& \widehat{T}_{b} \text { boundary shear stress divided by the density; } \\
& D \text { mean layer thickness for both layers; } \\
& H \text { total depth equal to } 2 D \text {; }
\end{aligned}
$$


reduced gravity, equal to $\left(\Delta \rho_{v} / \rho_{0}\right) g$; non-dimensional vertical density difference, approximately 0.025 ; mean density.

Carets have been used to indicate dimensional variables. The barotropic wave equation in $h$ is formed by differentiating (1a) with respect to $x$ and (1b) with respect to $t$ and subtracting. An analogous procedure involving $(2 a)$ and $(2 b)$ and $(3 a)$ and $(3 b)$ gives an internal wave equation in the lower layer elevation deviation $\widehat{h}_{2}$ :

$$
\begin{aligned}
g^{\prime} D \frac{\partial^{2} \widehat{h}_{2}}{\partial \widehat{x}^{2}}-2 & \frac{\partial^{2} \widehat{h}_{2}}{\partial \widehat{t}^{2}}+\frac{\partial}{\partial \widehat{x}}\left(\widehat{T}_{b}-2 \widehat{T}_{i}\right) \\
= & -\frac{g^{\prime}}{2} \frac{\partial^{2}\left(\widehat{h}_{2}\right)^{2}}{\partial \widehat{x}^{2}}-\frac{\partial^{2} \widehat{h}}{\partial \widehat{t}^{2}} \\
& -\left[\frac{1}{D} \frac{\partial^{2}\left(\widehat{Q}_{2}^{2}\right)}{\partial \widehat{x}^{2}}-\frac{1}{D} \frac{\partial^{2}}{\partial \widehat{x}^{2}}\left(\widehat{Q}-\widehat{Q}_{2}\right)^{2}\right]
\end{aligned}
$$

The fact that $D$ is $H / 2$ has been used repeatedly in (4). The equilibrium layer depth $D$ rather than $D+\widehat{h}_{2}$ appears in the convective acceleration and friction terms in (4), because the nonlinear contribution to these terms arising from the interface deviation is second order (and is thus neglected) in the perturbation analysis that follows. The full nonlinearity of the pressure gradient has, however, been retained in (4), as it will contribute in an important way to the residual flow.

Further development of these wave equations requires an approximation to the boundary and interfacial stress terms. The third-order Chebyshchev polynomial expansion of Dronkers [1964] has been used for this purpose. This expression has the advantage of providing for both tidal and mean flow contributions to the stress. It is most accurate at the extreme values (peak flood and ebb) when the stress is most important in the momentum balance. The bed and interfacial stresses (in terms of nondimensional velocity) are given by:

$$
\begin{gathered}
\widehat{T}_{b}=c_{d}\left|\widehat{U}_{2}\right| \widehat{U}_{2} \cong \frac{c_{d}}{\pi} \mathcal{U}_{i}^{2}\left(p_{1} U_{2}+p_{2} U_{2}^{2}+p_{3} U_{2}^{3}\right) \\
\widehat{T}_{i}=c_{i}\left|\widehat{U}_{2}\right|\left(\widehat{U}_{1}-\widehat{U}_{2}\right) \cong \frac{c_{i}}{\pi} \mathcal{U}_{i}^{2}\left[p_{1}\left(U_{1}-U_{2}\right)\right. \\
\left.+p_{2}\left(U_{1}-U_{2}\right)^{2}+p_{3}\left(U_{1}-U_{2}\right)^{3}\right]
\end{gathered}
$$

where $\mathcal{U}_{i}$ is the scale velocity for the baroclinic flow, $c_{d} \approx 2.5 \times 10^{-3}$ is the drag coefficient, $c_{i} \approx 0.5$ to $1 \times 10^{-3}$ is the interfacial exchange coefficient. The coefficients $p_{i}$ in $(5 a)$ and $(5 b)$ are functions of the ratio of river flow to tidal flow $\left(\widehat{Q}_{R} / \widehat{Q}_{t}\right)$. There is no river flow in the lower layer, and the $O\left(\epsilon_{i}\right)$ residual flow is small. Under these conditions, terms containing $p_{2}$ can be neglected in the bed stress, and $p_{1}$ and $p_{3}$ approach asymptotic values for $\widehat{Q}_{R} / \widehat{Q}_{t}=0$ of $8 / 3$ and $8 / 15$, respectively. The value of $c_{d}$ employed is within the usual range. The choice of $c_{i}$ was constrained to achieve consistency with our turbulence closure, the internal mixing calculation described be- low. Specifically, the interfacial thickness calculated from the shear and lower layer thickness implies a vertical momentum transfer. This momentum transfer, which varies from zero at times when the interface was not thickening to a maximum near peak ebb, was compared to the value determined from $c_{i}$ and the amplitude of the shear calculated from the harmonic, internal tide model derived from (4). The harmonic method does not allow global agreement throughout the tidal cycle to be achieved with a constant $c_{i}$. Instead, $c_{i}$ was adjusted until approximate agreement between the two peak flood values of vertical momentum transfer was achieved. Agreement at peak flood is appropriate, because the flood was found to be the critical time during the tidal cycle for the evolution of the density field and the residual flow (section 4 and part 2).

The internal tidal wave equation (4) and its barotropic analog may then be expanded in regular perturbation expansions. The barotropic nonlinearities are scaled by $\epsilon_{b}=\zeta / H$ (the ratio of barotropic tidal amplitude to average depth) as first used by Stokes [1847] and applied by Ianniello $[1977 a, b ; 1979 ; 1981]$ to the barotropic residual flow problem. The scaling of the vertically integrated barotropic continuity equation $(1 b)$ shows that the barotropic tidal velocity scale $\mathcal{U}_{b}$ is equal to $c \epsilon_{b}$, where $c$ is the barotropic wave celerity. The ratio $\epsilon_{b}=\zeta / H$ is then a Froude number indicating the stability of the barotropic oscillation. Although the dispersion parameter $(H / \lambda)^{2}$ (where $\lambda$ is the barotropic wavelength) is small enough that nonlinear dispersive effects may be ignored, these long waves are weakly dispersive because of the linear effects of friction in the $O(1)$ wave equation.

The analogous baroclinic parameter is $\epsilon_{i}=d / D$, the ratio of internal tidal amplitude to tidal cycle mean layer depth. This is a measure of the nonlinearity of the flow arising from the tidal cycle excursion of the density field. It provides the requisite behavior that the internal nonlinear effects increase with increasing tidal range up to the point where the tidal forcing is so strong that a two-layer structure can no longer be maintained. It is equal to $\mathcal{U}_{i} / c^{\prime}$ (where $\mathcal{U}_{i}$ is the internal wave particle velocity scale and $c^{\prime}$ is the internal wave speed), the internal scaling Froude number indicating the stability of the baroclinic oscillation. In most shallow estuaries, $\zeta / H$ is $O(0.01$ to 0.3$)$, the barotropic tidal wave is subcritical, and barotropic nonlinear effects remain small. The barotropic nonlinearities become $O(1)$ and bring about tidal bores only in exceptional cases. In contrast, $d / D$ ranges from perhaps 0.1 to a potential upper limit of 1 , and the nonlinear effects in the two-layer case are larger than those in the weakly stratified case. However, as is demonstrated in section 4, the two-layer circulation becomes unstable and breaks down well before $d / D$ reaches one.

It is also vital in a strongly reflective system such as the internal tidal flow analyzed here to distinguish between tidal amplitude as measured by $\epsilon_{i}=d / D$ and the actual excursion of the interface. Reference to the analogous barotropic problem - quarter wave resonance - is appropriate. It is well known that even an infinitesimal tide at the entrance of a system that is about one quarter of a wavelength long will be strongly amplified. The theoretically infinite response at the reflective barrier in 
an inviscid surface tide is rendered finite by any nonzero value of $c_{d}$, and increasing $c_{d}$ decreases the amplification [Dronkers, 1964]. Just as with the interface excursion near the head of a salt wedge, however, the actual surface excursion near the reflecting (landward) boundary may become large relative to the depth, as in the Bay of Fundy. This large excursion does not invalidate a perturbation analysis of reflective systems, because the actual wave amplitude scaled in the perturbation parameter is small, as is the transport near the reflecting boundary. It is, in fact, the smallness of the transport terms near the reflecting boundary that allows the wave equation derived via a perturbation expansion to remain valid. The perturbation expansion used here, therefore, is correct, despite the substantial interface excursion, for two-layer systems up to the point where the neap-spring transition occurs. It should also be noted that a small value of the scaling internal Froude number $\epsilon_{i}$ (as required for the validity of the perturbation expansion employed herein) is consistent with the observed [Geyer, 1985, 1988] total internal Froude number near 1 at the wedge head, because this total internal Froude number is strongly influenced by barotropic advection, without which the salt wedge advance and retreat would not be possible.

Energy transfer from the driving diurnal and semidiurnal tidal frequencies to higher and lower frequencies is one of the basic features of estuarine circulation. Tidal forcing at a single frequency leads to a zero frequency circulation, a tidal flow, and an overtide at $O(\epsilon)$. The residual, tidal, and overtide frequencies may be included by expanding the velocity and other variables in an asymptotic series, each term of which is further expanded in a Fourier series. Velocity and height variations at residual and overtide frequencies are excluded in $O(1)$, because the nonlinearly driven circulation at these frequencies is no larger than $O(\epsilon)$. Thus

$$
\begin{aligned}
\widehat{Q}_{i}={ }^{1} \widehat{Q}_{i}^{0}+\epsilon\left({ }^{0} \widehat{Q}_{i}^{1}+{ }^{1} \widehat{Q}_{i}^{1}+{ }^{2} \widehat{Q}_{i}^{1}\right) \\
+O\left(\epsilon_{i}^{2}\right) \quad i=1,2 \\
\widehat{Q}={ }^{1} \widehat{Q}^{0}+\epsilon\left({ }^{0} \widehat{Q}^{1}+{ }^{1} \widehat{Q}^{1}+{ }^{2} \widehat{Q}^{1}\right)+O\left(\epsilon^{2}\right)
\end{aligned}
$$

and similarly for the elevation variables. A subscript in (6) indicates the layer for a baroclinic flow variable; no subscript indicates a barotropic flow variable. The left superscript indicates the frequency ( 0 for mean, 1 for tidal, 2 for overtide), and the right superscript indicates the order of the term. Because the purpose of this analysis is to examine the generation of the mean or residual flow by the $O(1)$ tidal flow, the $O(\epsilon)$ overtide and $O(\epsilon)$ tidal frequency equations are not discussed here.

The scaling for the barotropic and baroclinic flows is as follows, with the numerical values of the scaling parameters taken to correspond to the situation in the Columbia River Estuary during the low-flow season:

$$
\begin{array}{ll}
\widehat{h}=\zeta h & \widehat{Q}=\mathcal{U}_{b} H Q \\
\widehat{z}=D z & \widehat{t}=\frac{t}{\omega} \\
\widehat{x}=L_{S} x & \widehat{T}_{i}=c_{d} \mathcal{U}_{i}^{2} T_{i} \\
\widehat{T}_{b}=c_{d} \mathcal{U}_{i}^{2} T_{b} & \widehat{Q}_{1}=\mathcal{U}_{i} D Q_{1} \\
\widehat{h}_{1}=d h_{1} & \widehat{Q}_{2}=\mathcal{U}_{i} D Q_{2}
\end{array}
$$

where

d interfacial excursion scale, equal to $2-3 \mathrm{~m}$;

$\omega$ the $M_{2}$ tidal frequency, equal to $1.4 \times 10^{-4}$

$L_{S}$ the salinity intrusion length scale, approximately $20-40 \times 10^{3} \mathrm{~m}$;

$\zeta$ the $M_{2}$ tidal amplitude scale, equal to $1 \mathrm{~m}$; $D \cong 8 \mathrm{~m}$.

The horizontal changes in baroclinic velocity are scaled under the assumption that along-channel changes in interface depth, not topographic features, are the dominant factors determining the convective accelerations. Thus the horizontal length scale for both layers is taken to be (Figure 9) the salinity intrusion scale length $L_{S}$, i.e., the distance that the salt wedge advances in half a tidal cycle. This was specified from observations for the model runs discussed herein. However, $L_{S}$ may also be related to the other scaling variables as $L_{S}=\pi / \omega\left(\mathcal{U}_{b}+\mathcal{U}_{i}-\widehat{U}_{R}\right)$, where $\widehat{U}_{R}$ is the river flow velocity. That is, the salt wedge will advance with the combined velocity of the barotropic and internal tides against the opposition of the river flow. This specification yields values of $L_{S}$ similar to observations.

The general validity of neglecting topographic variations was addressed in section 1; a more specific scaling argument can also be adduced. Consider lower layer mass conservation (equation $3 b$ )). Current meter power spectra [Jay, 1984] provide ample evidence that flow is confined to a few tidal and overtide frequencies. If only tidal frequency motion is considered, then the magnitude of $\partial \widehat{h}_{2} / \partial \widehat{t}$ fixes that of $\partial \widehat{Q}_{2} / \partial \widehat{x}$, independent of any variation of the $x$ length scale. If a topographic $x$ length scale $l_{t}<L_{S}$ were to be introduced, then the velocity scale associated with this length scale would have to be reduced (relative to the basic internal velocity scale) so that the magnitude of $\partial \widehat{Q}_{2} / \partial \widehat{x}$ remained invariant. Using this scaling, the convective accelerations (the wave equation term most likely to be influenced by topography) remain $O\left(\epsilon_{i}\right)$ up to the point that topographic changes become so sharp as to force division of the model into segments, each of which is, at least in barotropic wave calculations, assumed to be of constant depth and width [Dronkers, 1964]. The above argument concerning the scale of $\partial \widehat{Q}_{2} / \partial \widehat{x}$ assumes, as do the vertically integrated equations $(1 a),(2 a)$, and $(3 a)$, that horizontal velocities in each layer are nearly uniform. The strong shear in the interface layer almost certainly causes the convective accelerations to be locally $O(1)$. This cannot, however, be true for the flow as a whole, because the overtide and residual flows driven by the convective accelerations are observed to be only $O(\epsilon)$. Thus it is correct to take the convective accelerations to be $O(\epsilon)$ in our perturbation analysis.

Substitution of (5) to (7) in (4) results in a lower layer wave equation containing terms of different orders and frequencies. The terms at any given order and frequency must balance. This requirement yields the following $O(1)$ tidal and $O(\epsilon)$ residual equations for the motion in a twolayer estuary:

$$
\begin{aligned}
\frac{\partial^{21} h_{2}^{0}}{\partial x^{2}} & -\frac{p_{1}}{\pi} \frac{f \omega L_{S}^{2}}{g^{\prime} D} \frac{\partial^{1} h_{2}^{0}}{\partial t} \\
& \cong \frac{p_{1}}{\pi} \frac{\zeta}{D} \frac{\mathcal{U}_{i}}{\mathcal{U}_{b}} \frac{f_{i} \omega L_{S}^{2}}{g^{\prime} D} \frac{\partial^{1} h^{0}}{\partial t}
\end{aligned}
$$




$$
\begin{aligned}
\frac{\partial^{2}{ }^{0} h_{2}^{1}}{\partial x^{2}} & =\frac{\mathcal{U}_{i} L_{S}}{g^{\prime} D}\left\{\frac{p_{1}}{\pi}\left[f \frac{\partial Q_{I S}}{\partial x}-f_{i}\left(\frac{H}{D}\right)^{2} \frac{\partial Q_{S}}{\partial x}\right]\right. \\
& +\frac{p_{3}}{\pi}\left[f \frac{\partial}{\partial x}\left(\overline{\left({ }^{1} Q_{2}^{0}\right)^{2}} Q_{I S}\right)-f_{i}\left(\frac{H}{D}\right)^{2}\right. \\
& \left.\left.\times \frac{\partial}{\partial x}\left(\overline{\left({ }^{1} Q^{0}\right)^{2}} Q_{S}\right)\right]\right\}-\frac{1}{2} \frac{\overline{\partial^{2}\left({ }^{1} h_{2}^{0}\right)^{2}}}{\partial x^{2}} \\
& +\frac{U_{i}^{2}}{g^{\prime} D}\left[\frac{\partial^{2} \overline{\left({ }^{1} Q^{0}\right)^{2}}}{\partial x^{2}}-2 \frac{\partial^{2}}{\partial x^{2}} \overline{\left({ }^{1} Q^{0}{ }^{1} Q_{2}^{0}\right)}\right]
\end{aligned}
$$

along with the continuity equations

$$
\begin{gathered}
\frac{\partial^{1} Q_{2}^{0}}{\partial x}+\frac{\partial^{1} h_{2}^{0}}{\partial t}=0 \\
\frac{\partial^{1} Q_{1}^{0}}{\partial x}+\frac{\partial\left({ }^{1} h^{0}+{ }^{1} h_{1}^{0}\right)}{\partial t}=0 \\
\frac{\partial^{0} Q^{1}}{\partial x}+\frac{\partial Q_{S}}{\partial x}=0 \quad{ }^{0} Q^{1}+Q_{S}=Q_{R} \\
\frac{\partial^{0} Q_{2}^{1}}{\partial x}+\frac{\partial Q_{I S}}{\partial x}=0 \quad{ }^{0} Q_{2}^{1}+Q_{I S}=0
\end{gathered}
$$

where

$Q_{I S}$ internal mass transport, equal to $\overline{{ }^{1} Q_{2}^{0}{ }^{1} h_{2}^{0}}$;

$Q_{S}$ Stokes drift, equal to $\overline{{ }^{1} Q^{0}{ }^{1} h^{0}}$;

$f=\left(f_{b}+(H / D)^{2} f_{i}\right)$ in $s^{-1}$;

$f_{i}=c_{i} U_{i} / D \simeq 1.0 \times 10^{-4} \mathrm{~s}^{-1}$

$f_{b}=c_{d} \mathcal{U}_{i} / D \simeq 2.5 \times 10^{-4} \mathrm{~s}^{-1}$.

nonlinear terms (e.g., the convective accelerations) in equation $(8 b)$ have both zero frequency and overtide parts. The zero frequency part of each nonlinear term has been indicated by an overbar. The equality of the layer scale depths and the fact that the layer scale depth is half the total depth has been used repeatedly in deriving $(8 a)$ and $(8 b)$.

The $O(1)$ wave equation ( $8 a$ ) contains two homogeneous terms and one forcing term; it describes a damped internal wave driven by the barotropic tide. It is similar to the internal wave problem first discussed by Stokes [1847], wherein the particular solution to the internal wave problem is proportional to the surface wave; Stokes, however, considered only the inviscid case. The homogeneous part of the wave equation is a balance between the buoyant restoring force and damping. The acceleration term $\left(\partial h^{2} / \partial t^{2}\right)$ does not appear because it is smaller than the stress by the ratio $f / \omega \sim 5$, as is also true in the barotropic case [LeBlond, 1978]. Inclusion of the acceleration term in $O(1)$ does not appreciably change the results, but greatly complicates the algebra.

The residual surface elevation results from nonlinearities in the friction, pressure gradient and convective accelerations terms. The Stokes drift $Q_{S}$ and the internal mass transport $Q_{I S}$ (identified by Dore [1970]) appear in $(8 b)$ as result of substitution for ${ }^{0} Q^{1}$ and ${ }^{0} Q_{2}^{1}$, using continuity equations $(9 b)$ and $(9 c)$. The residual continuity equation suggests that the inward internal mass transport in the lower layer $Q_{I S}$ must be compensated by a zerofrequency, Eulerian outflow in the bottom layer. The sum of the continuity equations for the two layers $((9 c)$ plus the analogous equation for the upper layer) is the continuity equation for the total or barotropic flow. Thus, the baroclinic nonlinearity exists simultaneously with and in addition to the barotropic nonlinearity.

\section{Solution of the Governing Equations}

It is appropriate in an infinite, shallow channel with uniform cross section and strong friction to assume that the barotropic tide consists of only an incoming wave, gradually damped in the positive- $x$ direction. The relevant solution [ Jay, 1987; LeBlond, 1978] for the $O(1)$ barotropic tide ${ }^{1} h^{0}$ is

$$
\begin{aligned}
{ }^{1} h^{0} & =\operatorname{Re}\left(M(x) e^{i t}\right) \\
{ }^{1} Q^{0} & =\operatorname{Re}\left(\frac{1+i}{4 \pi} M(x) e^{i t}\right) \\
M(x) & =H_{0} e^{-2 \pi(1+i) k_{b} L_{S} x}
\end{aligned}
$$

where $k_{b}=1 /(2 \pi)\left[p_{1} f_{b} \omega /(g H)\right]^{1 / 2}$ is the barotropic wave number. The real and imaginary parts of the wave number for a uniform channel are equal, in the high-friction limit; this is the result of the dominance of the friction term over the acceleration term in $(8 a)$. The barotropic wavelength $1 / k_{b}$ is much larger than $L_{S}$, so that the barotropic wave changes phase and is damped very little in the estuary.

The internal tidal oscillation, the solution to $(8 a)$ and $(9 a)$, is

$$
\begin{array}{r}
{ }^{1} h_{2}^{0}=\operatorname{Re}\left[(N-K M) e^{i t}\right] \\
{ }^{1} Q_{2}^{0}=\operatorname{Re}\left[\frac{-\omega L_{S}}{\mathcal{U}_{i}}\left(\frac{N^{\prime}}{K_{4}}-\frac{K}{K_{2}} M^{\prime}\right) e^{i t}\right] \\
N=A e^{2 \pi(1+i) k L_{S} x}+B e^{-2 \pi(1+i) k L_{S} x}
\end{array}
$$

where $k=\left[p_{1} /\left(8 \pi^{3}\right) f \omega /\left(g^{\prime} D\right)\right]^{1 / 2}$ is the baroclinic wave number,

$$
\begin{aligned}
K_{2} & =8\left(\pi k_{b} L_{S}\right)^{2} \\
K_{4} & =8\left(\pi k L_{S}\right)^{2} \\
K & =\frac{\zeta}{D} \frac{u_{i}}{u_{b}} \frac{f_{i} \omega p_{1}}{\left(8 \pi^{3} g^{\prime} D k_{b}^{2}-p_{1} f \omega\right)}
\end{aligned}
$$

The baroclinic tidal solution consists of incoming and reflected waves, plus a forced term in response to the barotropic tide. The amplitude of the reflected wave relative to the incoming wave is determined by a reflective boundary condition below. The homogeneous part of the solution dominates ${ }^{1} h_{2}^{0}$; that is, the baroclinic part of the interfacial excursion is greater than the component driven by ${ }^{1} h^{0}$. The homogeneous solution to $(8 a)$ is dispersive - the internal wave speeds increase weakly with increasing frequency. Similar results could have been achieved by imposing a rigid lid on the baroclinic problem and then adding the barotropic and baroclinic solutions. This would have slightly degraded the accuracy of the calcu- 
lated interfacial elevations, but more importantly, it would have obscured the basic dynamics of the problem. The proportionality constant between the barotropic wave and the baroclinic response thereto is denoted by $K$. In an infinite inviscid two-layer system, $K=-1 / 2$, so that half the barotropic transport occurs in the lower layer. The finite length of the lower layer and presence of friction change, however, both the amplitude and phase of $K$.

The $O(\epsilon)$ residual solution may be found by integrating (8b) twice:

$$
\begin{aligned}
& { }^{0} h_{2}^{1} \cong C_{1} x+C_{2}-\frac{1}{2} \overline{\left({ }^{1} h_{2}^{0}\right)^{2}} \\
& +\frac{\mathcal{U}_{i} L_{S}}{g^{\prime} D}\left[\left(\frac{p_{1}}{\pi}+\frac{p_{3}}{\pi} \overline{\left({ }^{1} Q_{2}^{0}\right)^{2}}\right) f \int Q_{I S} d x\right. \\
& -f_{i}\left(\frac{H}{D}\right)^{2}\left(\frac{p_{1}}{\pi} \int Q_{S} d x\right. \\
& \left.\left.+\frac{p_{3}}{\pi} \int \overline{\left({ }^{1} Q^{0}\right)^{2}} Q_{S} d x\right)\right] \\
& Q_{S}=\frac{|M|^{2}}{8 \pi} \\
& \int Q_{S} d x=-\frac{|M|^{2}}{32 k_{b} L_{S} \pi^{2}} \\
& \int \overline{\left({ }^{1} Q^{0}\right)^{2}} Q_{S} d x=-\frac{|M|^{4}}{64 k_{b} L_{S} \pi^{2}} \\
& Q_{I S}=-{ }^{0} Q_{2}^{1}=\frac{\omega L_{S}}{2 \mathcal{U}_{i}} \operatorname{Re}\left(\frac{K^{2}}{4 \pi k_{b} L_{S}}|M|^{2}\right. \\
& \left.+\frac{K}{K_{4}} N^{\prime} M^{*}+\frac{K}{K_{2}} N^{*} M^{\prime}-\frac{N^{*} N^{\prime}}{K_{4}}\right) \\
& \int Q_{I S} d x=\frac{-\omega L_{S}}{2 \mathcal{U}_{i}} \operatorname{Re}\left[\frac{|N|^{2}}{2 K_{4}}+\frac{K^{2}}{2 K_{2}}|M|^{2}\right. \\
& \left.-\frac{K}{K_{2}+K_{4}} K_{5}\left(i M^{\prime *} N^{\prime}+2 M N^{*}\right)\right] \\
& \overline{\left({ }^{1} Q_{2}^{0}\right)^{2}}=\frac{1}{2}\left(\frac{\omega L_{S}}{\mathcal{U}_{i}}\right)^{2}\left[\frac{\left|N^{\prime}\right|^{2}}{K_{4}^{2}}\right. \\
& \left.+\left(\frac{K}{K_{2}}\right)^{2}\left|M^{\prime}\right|^{2}-\frac{2 K}{K_{2} K_{4}} R_{e}\left(M^{\prime} N^{\prime *}\right)\right]
\end{aligned}
$$

where

$$
K_{5}=K_{4}^{-1}-K_{2}^{-1}
$$

The residual surface slope is dominated by its homogeneous part $\left(C_{1} x+C_{2}\right)$, the integral of the internal mass transport $Q_{I S}$ and the nonlinear surface slope forcing. The identity

$$
\overline{R_{1} R_{2}}=\frac{1}{2} \operatorname{Re}\left(r_{1} r_{2}^{*}\right)
$$

where

$$
R_{1}=r_{1} e^{i t} \quad R_{2}=r_{2} e^{i t}
$$

and repeated integration by parts have been used in evaluating the integrals in $(12 a)-(12 g)$. One further simplification was made: $\overline{\left({ }^{1} Q_{2}^{0}\right)^{2}}$ was taken out of the first integral proportional to $p_{3}$ because the dominant term in $\overline{\left({ }^{1} Q_{2}^{0}\right)^{2}}$ comes from the barotropic tide, which from (10) has a very long wavelength relative to the salinity intrusion length.

\section{Application of Boundary Conditions}

Application of the boundary conditions is the most complex part of the analytical model. Reference to the analogous barotropic tidal problem in a channel of constant width and depth is useful in understanding the conditions applied here. The tidal long-wave differential equation is second order and requires two boundary conditions to determine the amplitudes and phases of the incident and reflected waves. Determination of both amplitude and phase for each wave means that the coefficient usually referred to as the wave amplitude is, in fact, a complex number. There are two possibilities for a surface tidal wave: either the wave is reflected by the landward boundary of the system (as in many bays), or the system is long relative to the damping length of the tidal wave and the wave dies out towards infinity in the positive- $x$ direction (as in tidal rivers). The first situation gives rise, in the absence of friction, to a standing wave, in which incident and reflected waves are of equal magnitude. If friction is present, the two waves have the same magnitude only at the point of reflection, but the rise and fall of the tide throughout the system is still nearly synchronous. In contrast, only an incident wave is present in a tidal river, and the tide is progressive; the time of high water varies strongly along the channel. The latter is the case with the barotropic tide in channel of constant width that drives the salt wedge flow discussed here. The salinity intrusion length $L_{S}$ is short, however, relative to the barotropic tidal wavelength and little progression of the barotropic wave is actually observed over a distance of $L_{S}$.

Because the internal wave speed is less than the surface wave speed, the internal wave length is smaller than the barotropic wave length, and $L_{S}$ is nearly one quarter of the baroclinic wavelength. That the motion of the interface is essentially simultaneous throughout this length [Geyer, 1985] requires that a reflected wave be present, and the advancing salt wedge is not a progressive wave, despite the movement of the salt wedge head. Confusion over whether the salt wedge is progressive or reflective arises primarily because wave problems are usually considered either in an infinite domain, or one in which fixed boundaries are present, not in a domain with a movable control section. Reflection at the head can also be understood in terms of the reduced thickness of the lower layer and reduced stability at the head, caused by the interfacial jet [Geyer, 1985, 1988] (see also section 2). The head must always propagate more slowly than the body of the salt wedge. Thus partial reflection is inevitable. In fact, because the inhomogeneous (barotropically forced) component of the internal tide moves much more rapidly than $c^{\prime}$ and dominates $\widehat{Q}_{2}$, reflection should be total, or nearly so. This agrees with the observed essentially simultaneous rise and fall of the interface throughout the system. 
Continuing with the barotropic analogy, the amplitude of the reflected surface wave relative to the incoming wave in a system of finite length is determined by requiring that the transport vanish at the landward boundary. The amplitude of the incident barotropic wave is then set by the phase and amplitude of the tide at the entrance. Two more conditions are required to determine the constants in the second-order barotropic residual flow equation; these, however, are less closely analogous to the internal case analyzed herein. Another important difference between the barotropic and salt wedge analyses is that the external Froude number of the barotropic tidal wave is normally quite small (of $O\left(\epsilon_{b}\right)$ ). In contrast, the flow at the head of the advancing salt wedge is internally critical. Application of a perturbation model to such a strongly nonlinear region is perhaps questionable, despite the distinction between $\epsilon_{i}$ and the total internal Froude number discussed earlier. The model does not, therefore, directly represent the complex physics of the wedge front. Rather, the boundary conditions are chosen to force approximately correct behavior of the head of the advancing and retreating wedge, as well as determine the constants in the analytical solution. The boundary conditions are applied to the total flow, here approximated by the sum of the $O(1)$ tidal and $O(\epsilon)$ residual components.

The boundary conditions for the salt wedge problem are

${ }^{1} Q_{2}^{0}(1, t)=0$ for all $t$

$h_{2}\left(0, t_{s e}\right)={ }^{1} h_{2}^{0}\left(0, t_{s e}\right)+{ }^{0} h_{2}^{1}(0) \cong 1.25$

$h_{2}\left(1, t_{s f}\right) \cong 0.7$

$F^{2}=F_{1}^{2}+F_{2}^{2}=1$ at $x=x_{f}, t=t_{f}$

the phases of ${ }^{1} Q^{0}$ and ${ }^{1} Q_{2}^{0}$ are identical at

$$
x=0
$$

where

$t_{s f}$ slack after flood;

$t_{s e}$ slack after ebb;

$t_{f}$ peak flood;

$F_{1}^{2}=\frac{u_{i}^{2}}{g^{T} D} \frac{\left({ }^{1} Q_{1}^{0}\left(x_{f}, t_{f}\right)+Q_{R}\right)^{2}}{\left(H-H_{2}\right)^{3}} ;$

$F_{2}^{2}=\frac{u_{i}^{2}}{g^{\prime} D} \frac{\left({ }^{1} Q_{2}^{0}\left(x_{f}, t_{f}\right)\right)^{2}}{H_{2}{ }^{3}}$

$F_{1}^{2}, F_{2}^{2}$ layer Froude numbers;

$x_{f}$ position of the head of the wedge at peak flood.

The first of these (equation $(14 a)$ ) is a reflective condition that defines the magnitude of the reflected wave relative to the incoming wave. The second and third conditions, $(14 b)$ and $(14 c)$, set the height of the wedge at the end of ebb and the end of flood, and are based on observations in the Columbia River Estuary. They determine $C_{1}$ and $C_{2}$ in the residual height (equation $(12 a)$ ) and impose a mean slope on the interface. They also require the interface to be somewhat above mid-depth at the entrance at the onset of flood and somewhat below mid-depth at $x=L_{S}$ at the end of flood. The "mouth" of the theoretical estuary is taken to be the position of the minimum salinity intrusion at the end of ebb. In both the Columbia River and Fraser River estuaries, this minimum occurs at or near the entrance bar. In other systems, the position of the minimum salinity intrusion might be determined by other factors.

The conditions of $(14 d)$ and (14e), like the entrance condition for the barotropic tide, set the amplitude and phase of the incoming internal wave. The internal Froude number condition requires the head of the wedge to be in an internally critical state. In fact, this condition is approximately satisfied during almost the entire flood [Geyer, $1985,1988]$, but one of the limitations of the harmonic form assumed for the solution is that the boundary condition can be exactly satisfied at only one time. This is rectified by the specification of the height of the front of the wedge in $(14 b)$ and $(14 c)$. Normally, the model produces a wedge that is apparently subcritical during most of its advance. In nature, the reduced stratification at the head of the wedge caused by interfacial advection and mixing at the head of the wedge keeps the head critical, even when the flow behind the wedge is subcritical. Thus the assumed critical front on flood is realistic. Behavior of the wedge on ebb is different than on flood in that the head of the wedge no longer acts as a movable control section. The lower layer is therefore allowed to collapse to zero thickness as it retreats. As the lower layer thins on ebb, the strong shear causes the flow to become supercritical, and strong mixing occurs. The case in which the wedge becomes supercritical throughout on flood and is destroyed by mixing during its advance corresponds to the neap-spring transition, and is discussed below.

The nonlinearity of the Froude number condition, the fact that the position at which this condition is applied $\left(x_{f}\right)$ is an unknown, the dependence of $C_{1}$ and $C_{2}$ on the properties of the incoming wave, and the ebb-flood difference in stratification mean that the entire boundary condition calculation is best done iteratively by computer.

\section{Modification of the Analytical Solution by Mixing}

The determination of the analytical solution and application of the boundary conditions described above assumes the $O(1)$ flow properties vary harmonically in time. Part of the ebb-flood asymmetry which generates the residual circulation emerges from this harmonic decomposition in the form of the correlations between tidal elevations and transports that constitute the Stokes drift $Q_{S}$ and internal mass transport $Q_{I S}$. Any representation of the ebb-flood asymmetry would be incomplete, however, without a calculation of vertical mixing. The unidirectional tendency during the tidal cycle toward increased mixing cannot readily be represented harmonically and must be calculated with a time-stepping scheme in time and space. The information required at each time includes the position of the head of the intrusion, and the interface elevation and the velocity in both layers as a function of $x$. It is assumed that the $O(1)$ and $O(\epsilon)$ flow variables for the two-layer flow (given by $(9 a),(9 c),(11)$, and (12)) apply at all points seaward of the position of the head of the wedge at each $t$, and that the barotropic properties of (9b) and (10) apply landward of the head of the wedge.

Determination of the interfacial thickness $\Delta \widehat{i}$ is based on two observations. First, Kelvin-Helmholtz instabilities occur in the Fraser River Estuary salt wedge whenever the total Richardson number $R i_{T}$ (based on the mean plus fluctuating shears) exceeds a critical value of about $1 / 4$. These instabilities cause the interface layer to thicken un- 
til $R i_{T}$ is reduced to the critical level [Geyer, 1985; Geyer and Smith, 1987]. Second, the velocity and salinity in the interfacial layer tend, as the thickness of the layer grows, toward a linear profile. The tendency toward linear interfacial profiles is particularly strong on ebb [Geyer and Smith, 1987]. On strong floods in both the Columbia River and Fraser River estuaries, an interfacial velocity maximum is observed, the dynamics of which cannot be so simply represented (e.g., Figure 7). Nonetheless, this jet is prominent only for part of the tidal cycle, and the primary purpose here is determination of the thickness of the interfacial layer, not its precise velocity structure. Thus a layer Richardson number approach may be used as a good first approximation in calculating interfacial properties.

To use a Richardson number criterion in a harmonic model, it is necessary to relate the Richardson number based on the total shear $R i_{T} \cong 0.25$ to $\overline{R i}$, the Richardson number based on the tidal and residual shear. The relationship between $R i_{T}$ and $\overline{R i}$ is (as modified slightly from Geyer and Smith, [1987]):

$$
0.25 \cong R i_{T}=\left(\frac{1}{\overline{R i}}+\frac{1}{R i^{\dagger}}+\frac{1}{R i+t}\right)^{-1}
$$

where $R i^{+}=g^{\prime} \Delta \widehat{i} /\left[\alpha\left(\widehat{U}_{2}\right)^{2}\right]$ is turbulent variance associated with the bottom boundary, $\alpha$ is an empirical constant of $O(0.1)$, and $R i^{++} \cong 1$ is the variance related to the saturated internal wave field. $R i_{T}$ in $(15 a)$ includes contributions to the total shear from the mean flow, from a high-frequency internal wave field in which kinetic and potential energy are partitioned equally, and from turbulence in the lower layer caused by the bottom boundary, as estimated by the empirical constant $\alpha \cong 0.1$. The surface layer is assumed inviscid. The value of $\overline{R i}$ is $1 / 3$ for $\alpha=0$ but $>1 / 3$ for $\alpha>0$.

The local interfacial layer thickness $\Delta \hat{i}$ may then be calculated for each $x$ and $t$ from $\overline{R i}$ and the shear $\Delta \widehat{U}$ :

$$
\Delta \widehat{i}=\overline{R i} \frac{\Delta \widehat{U}^{2}}{g^{\prime}}
$$

The presence of the interface changes the speed of the fluid in the remainder of the each layer; thus, the mean shear $\Delta \widehat{U}$ depends on $\Delta \hat{i}$. Then $(15 b)$ may be rewritten as a cubic polynomial equation for $\Delta \hat{i}$. If no real root less than twice the layer depth of the shallower of the two layers exists, then the wedge has been destroyed at that $\widehat{x}$ and $\widehat{t}$. Once the two-layer system has been destroyed, only the barotropic wave equation need be satisfied at that position. Uniform flow is then assumed on the flood and linear shear on the ebb, in accordance with observations (e.g., Figures 6 to 8 ) and the ebb-flood asymmetry in the interaction of the barotropic and baroclinic pressure gradients. Mixing driven by the Kelvin-Helmholtz instabilities is presumed to continue even after the interface reaches the depth of flow. The result on ebb is a reduction of stratification until the $\overline{R i}$ criterion is met, given the ambient shear. The assumption of uniform flow on flood requires the assumption of a uniform density field as well.

Mixing is irreversible, so it would be unreasonable to allow the interface thickness to decrease during the wan- ing parts of the flood and ebb. Thus, once the $\Delta \widehat{i}$ field has been determined at a given time, each $\Delta \widehat{i}$ is compared to the greatest previous value for that distance behind the head of the intrusion. The greater of the two values is the actual interface thickness. Calculation of the interfacial properties for the two-layer part of the system completes the determination of the density and velocity fields for the tidal cycle. Flow is assumed to be vertically uniform above and below the interfacial layer and linear in the interface. A bookkeeping routine then determines the tidal cycle average properties. An entire tidal cycle with a $\Delta \hat{t}$ of 0.25 tidal hour requires less than $2 \mathrm{~min}$. on a VAX $11 / 780$.

\section{Results and Discussion: Properties of Shallow, Two-Layer Tidal Flows}

\section{Tidal Properties}

Basic properties of the model response for the low flow season were calculated using a river flow of $\left(\widehat{Q}_{R}\right)$ of 0.1 and $0.15 \mathrm{~m} \mathrm{~s}^{-1}$ and a tidal amplitude $\left(H_{0}\right)$ of 0.2 to $2 \mathrm{~m}$. Increasing the tidal range or river flow decreases the amplitude of the internal oscillation because of the increased friction. The resulting analytical solutions predict the amplitude of ${ }^{1} h_{2}^{0}$ to be in the range of $\sim 0.15$ to $\sim 0.6$, with typical values of 0.2 to 0.3 . These typical values are near the upper limit of validity of the perturbation method used (section 3), while the larger values of ${ }^{1} h_{2}^{0}$ are not meaningful because they always result in destruction of the two layer flow in the subsequent mixing calculation, i.e., transition to another flow regime. Thus the perturbation method is valid under the conditions in which it was employed.

Typical late flood and ebb velocity and salinity sections for low-flow, neap tide conditions are shown in Figures $10 a$ to $10 d$. The highest velocities are found in each layer where the layer is thin - at the entrance in the upper layer and near the nose in the lower layer. The interfacial thickness is small everywhere on flood (Figures $10 a$ and $10 b$ ), because of the relatively weak shear. The late ebb section (Figure $10 c$ and $10 d$ ) shows the effects of collapse of the wedge. Early in the ebb, mixing begins at the entrance, where velocities are large, and at the nose of the wedge, where the lower layer is thin. By late ebb, active mixing has extended throughout the entire length of the saline intrusion, yielding linear velocity and density fields throughout.

\section{Ebb-Flood Asymmetry and Generation of the Residual Circulation}

The predicted residual Eulerian velocity and salinity fields for the low-flow, neap tide of October 16-17, 1980 are shown in Figure $11 a$ and $11 b$. Results from 50-hour averages at 12 Aanderaa current meters are shown for comparison. The model predicts net upstream bottom flow in the seaward 0.55 of the channel and weak seaward flow elsewhere at the bottom (Figure 11b). Because slip is allowed, the maximum inflow of $0.22 \mathrm{~m} \mathrm{~s}^{-1}$ occurs at the bed and is almost twice the maximum observed value of $0.12 \mathrm{~m} \mathrm{~s}^{-1}$. Observations show net upstream bottom flow at only three meters - two near the entrance and one in a deep hole near $x=0.66$. Outflow is strong at both meters at $x=0.1$. These two meters were located close to the south side of the estuary to avoid conflict with ship- 

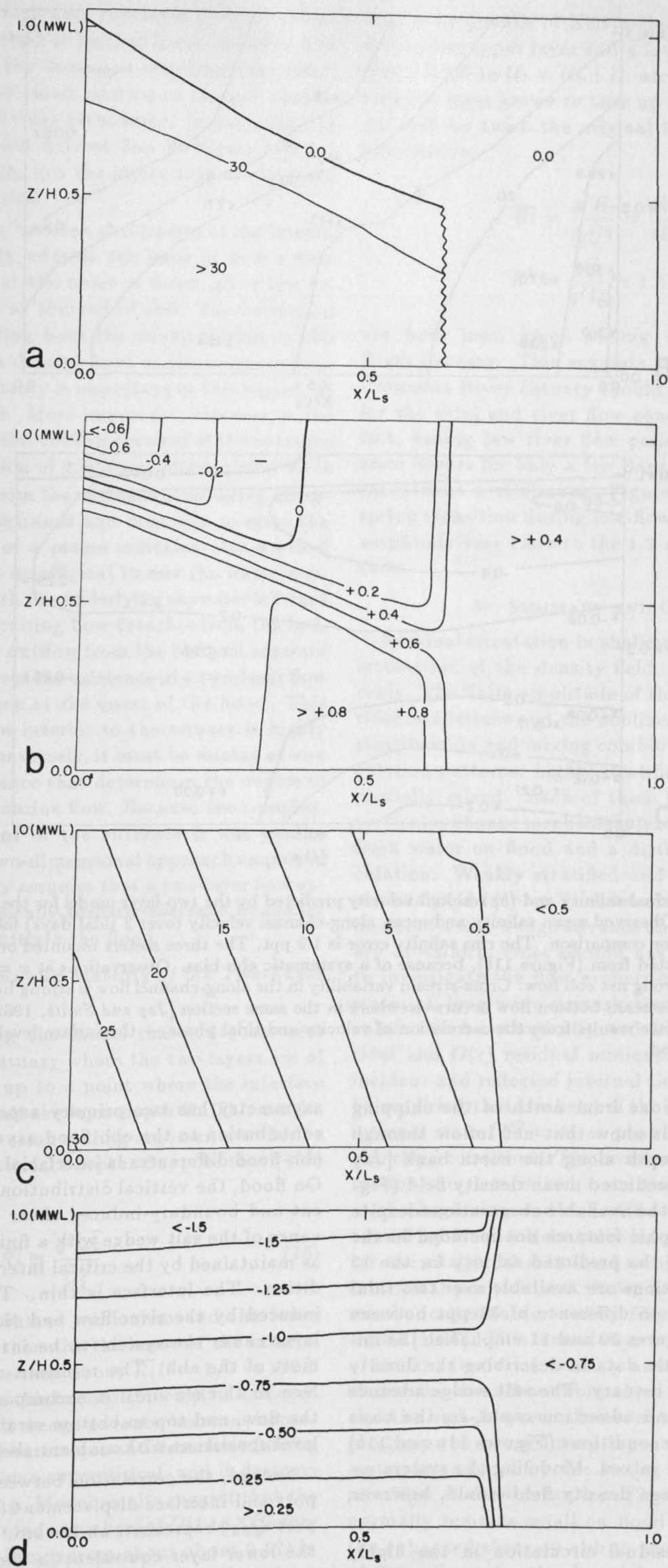

Fig. 10. Two-layer layer model predictions of $(a)$ salinity in parts per thousand and $(b)$ velocity in meters per second for late flood and of $(c)$ salinity and $(d)$ velocity for late ebb for a tidal amplitude of $1 \mathrm{~m}$ and a river flow of $0.15 \mathrm{~m} \mathrm{~s}^{-1}$ (Figure 10a). This approximates fall, low-flow, neap tide conditions. 

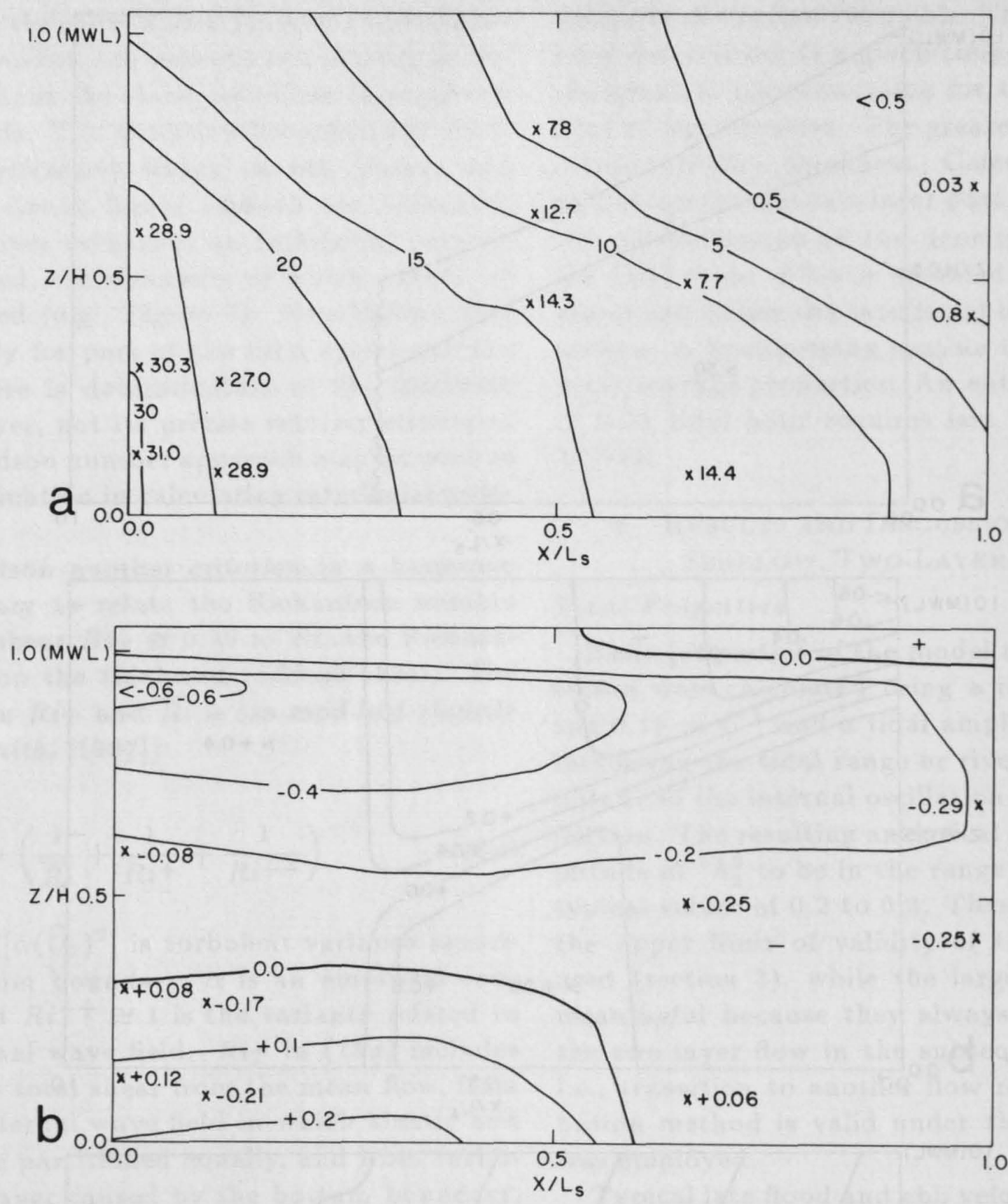

Fig. 11. (a) Residual salinity and (b) residual velocity predicted by the two-layer model for the same conditions as in Figure 10. Observed mean salinity and mean along-channel velocity (over 2 tidal days) for October 16-17, 1981 , are shown for comparison. The rms salinity error is $1.9 \mathrm{ppt}$. The three meters mounted on a bridge-pier at $x=0.47$ are omitted from (Figure $11 b$ ), because of a systematic ebb bias. Observations at $x=0.09$ are from a location with a strong net ebb flow. Cross-stream variability in the along-channel flow is strong because of channel curvature, and upstream bottom flow occurs elsewhere in the same section [Jay and Smith, 1989]. Net upstream flow near the surface results from the correlation of velocity and tidal phase - the surface level is above MLLW mainly on the flood.

ping. Current observations from north of the shipping channel for other periods show that net inflow through this section occurs at depth along the north bank [Jay and Smith, 1989]. The predicted mean density field (Figure 11a) closely matches the available observations despite the influence of topographic features not included in the model. The rms error of the predicted salinity for the 12 points at which observations are available over two tidal days is $1.9 \mathrm{ppt}$, relative to difference of $33 \mathrm{ppt}$ between fresh and salt water. Figures 10 and 11 emphasize the importance of using synoptic data in describing the density field or circulation in an estuary. The salt wedge advance and retreat by mixing and advection could, on the basis of the tidal cycle average conditions (Figures $11 a$ and $11 b$ ) be described as partially mixed. Modeling the system using the tidal cycle average density field would, however, be quite misleading.

Generation of the residual circulation in the highly stratified case is best viewed as resulting not from the interaction of the mean circulation and density field, but primarily from the ebb-flood asymmetry. The ebb-flood asymmetry has two primary aspects. The largest single contribution to the ebb-flood asymmetry comes from the ebb-flood differences in interfacial elevation and thickness. On flood, the vertical distribution of total pressure gradient and boundary-induced shear cause the wave like advance of the salt wedge with a finite thickness at its head, as maintained by the critical internal Froude number condition. The interface is thin. The enhanced ebb shear induced by the river flow and the thinning of the lower layer cause the system to be internally supercritical for most of the ebb. The resultant mixing causes the interface to thicken until it encompasses the entire depth of the flow, and top-to-bottom stratification is reduced to a level consistent with ambient shear.

Second, the correlation between the lower layer transport and interface displacement (the internal mass transport $\widehat{Q}_{I S}$ ) represents an inward, Lagrangian transport in the lower layer equivalent in the neap tide case to 0.03 to $0.1 \mathrm{~m} \mathrm{~s}^{-1}$. This must be compensated by an equal zero-frequency Eulerian outflow in the lower layer. How this Eulerian outflow is distributed within the lower layer 
cannot be predicted with this two-layer model; a zerofrequency inflow very close to the bed is not excluded, but such an inflow is not the dominant zero-frequency effect and would certainly be small relative to the net circulation arising out of the tidal asymmetry. In any case, the substantial net upstream bottom flow in Figure $11 b$ is a result of tidal processes, not the interaction of the mean velocity and density fields.

The model does not, because of the form of the boundary conditions, directly address the issue of how a twolayer flow is initiated at the onset of flood, given the destruction of the layers at the end of ebb. The horizontal density gradient resulting from the strong mixing on ebb and the formation of a density front at the entrance (evident in Figure $5 b$ ) probably is important in this regard, as one reviewer suggested. More important, however, is the lack of confinement of the outflow seaward of the entrance section and the formation of a buoyant plume there. Even if most of the outflow from the entrance is advected alongshore away from the entrance and does not re-enter the estuary, the existence of a plume indicates that vertical momentum exchange is insufficient to mix the water leaving at the end of ebb with the underlying seawater seaward of the point where the exiting flow detaches from the bed. This detachment of the outflow from the bed just seaward of the entrance guarantees the existence of a two-layer flow just outside the entrance at the onset of the flood. This is true whether the flow interior to the estuary is highly or weakly stratified. Conversely, it must be mixing at and inside the estuary entrance that determines the degree of stratification of the estuarine flow. Because the complex, unconfined flow seaward of the entrance is not readily represented using the two-dimensional approach employed herein, the model simply assumes that a two-layer flow exists and employs a physically realistic entrance boundary condition to correctly initiate the flood.

Neap-Spring Transitions and Stability of the Two-Layer System

It was found in using the model that (15b) has real roots (at least in mid-estuary where the two layers are of similar thickness) only up to a point where the interface thickness $\Delta \hat{i}$ is about half the layer depth $D$. This can be explained physically by using scaling arguments to derive an upper limit on the interface depth. Suppose that $(15 b)$ is approximated by

$$
\overline{R i} \cong \frac{g^{\prime} \Delta \widehat{i}}{\widehat{U}_{2}^{2}}
$$

If the upper layer is assumed motionless and the lower layer critical $\left(F_{2}=1\right)$, then the thickness of the interfacial layer cannot be more than about $\overline{R i} D=1 / 3$ to $1 / 2 D$ depending on $\alpha$. Greater thicknesses can only be achieved with $F_{2}>1$. Thus, a salt wedge advance becomes impossible when the combined barotropic and baroclinic forcing causes the wedge to become supercritical, and it destroys itself by interfacial mixing. Model results suggest that the wedge reaches internal Froude numbers of $O(1$ to 3$)$ before the interfacial thickness jumps from about about $0.5 \mathrm{D}$ in mid-estuary to the depth of flow as $(15 b)$ ceases to have real roots. This agrees with the flume studies of Lawrence [1985] that show the actual mechanism of layer destruc- tion to be growth of Kelvin-Helmholtz instabilities. If a motionless upper layer and a lower layer with an advance speed equal to $\mathcal{U}_{I}=\mathcal{U}_{b}+\mathcal{U}_{i}$ are assumed, then as the interfacial layer grows to take up the entire flow, the shear $\Delta \widehat{U}$ will be twice the original lower layer velocity. The two criteria,

$$
\begin{aligned}
& \overline{R i}=\frac{g^{\prime} H}{\Delta \widehat{U}^{2}}=\frac{g^{\prime} H}{4 \mathcal{U}_{I}^{2}} \cong 0.35 \\
& F^{2}=\frac{\mathcal{U}_{I}^{2}}{g^{\prime} D} \cong 1.5
\end{aligned}
$$

are both met, given scaling values for the Columbia River Estuary. This suggests that the salt wedge in the Columbia River Estuary should be only marginally stable for the tidal and river flow conditions modeled here. In fact, during low river flow periods, the highly stratified state occurs for only a few days at a time when the tidal range is at a minimum. Figure 1 shows that the neapspring transition during low-flow periods occurs at a tidal amplitude very close to the $1.2 \mathrm{~m}$ suggested by model results.

\section{Summary and Conclusions}

Residual circulation in shallow estuaries arises from the interaction of the density field, river flow and tidal currents. The finite amplitude of the barotropic and internal tidal oscillations and the nonlinearity of the interaction of stratification and mixing combine to create three tidal circulation patterns: highly stratified, weakly stratified, and partially mixed. Each of these has a different dominant vertical exchange mechanism leading to mixing of salt and fresh water on flood and a distinct type of residual circulation. Weakly stratified and partially mixed systems are analyzed in part 2. A two-layer, harmonic analytical model of a salt wedge advance and retreat in shallow estuary of uniform cross section has been constructed herein to investigate the flow properties in the highly stratified state. A long-wave perturbation method is used to calculate the barotropically and baroclinically forced $O(1)$ tidal and $O(\epsilon)$ residual motions. The amplitudes of the incident and reflected internal tidal waves are determined by requiring that the head of the salt wedge be in a critical internal state $\left(F^{2}=1\right)$ at peak flood and by imposing a reflective condition. The constants in the zero-frequency motion are determined from the elevation of the wedge at the beginning and end of flood. Interfacial thickening caused by Kelvin-Helmholtz instabilities is calculated after the analytical solution has been determined from the interfacial position and shear, using the bulk Richardson number criterion derived by Geyer [1985] and Geyer and Smith [1987]. This formulation is based on the assumption of linear interfacial velocity and density profiles and includes the shear from both the deterministic tidal motion and the high-frequency internal wave field. The model reproduces the two primary aspects of the ebb-flood asymmetry introduced by the strong buoyancy flux in highly stratified shallow estuaries. First, the interface thickness normally remains small on flood but expands because of the enhanced shear on ebb to encompass the entire depth of flow. Second, a landward Lagrangian drift associated with the finite amplitude of the internal tide is compensated by an outward Eulerian flow. 
The model reproduces the tidal and residual velocity and density fields observed under low-flow conditions in the Columbia River Estuary with reasonable fidelity and correctly predicts that the mean shear is much greater in the highly stratified case than in the weakly stratified and partially mixed flows considered in part 2. Model results and theoretical arguments show that the internal hydraulic state on the flood is the critical factor in determining whether or not an internal wavelike advance in a highly stratified system is possible. If the tidal range is large enough that the advancing salt wedge is unstable on flood, then the interfacial layer expands to the depth of flow, and the two-layer system is destroyed. This is the neap-spring transition. Either $\overline{R i}$ or $F$ in (17) may be used as an indicator of the stability of the saline intrusion; modified forms of $F$ are used both in the discussion of the partially mixed state (Part II) and a new estuary classification system [ Jay and Smith, 1988]. Increases in tidal range and decreases in river flow tend to destabilize the highly stratified flow. The timing of neap-spring transitions in a real system may also be influenced by factors not included in the model, for instance, topography features and the diurnal inequality.

The present model does not consider the response of a highly stratified flow to topography. This has been justified by qualitative arguments that suggest that topographic features are not the primary factor determining the character of the baroclinic circulation in shallow estuaries, by scaling arguments and a posteriori by the success of the model in predicting circulation patterns and neap-spring transitions. Nonetheless, observations [Geyer, 1985, 1988] have shown that because of the nearcritical internal hydraulic state of the salt wedge head, such systems show a substantial response of interfacial position and thickness to bars, constrictions, and depressions in the channel. The ability of the present weakly nonlinear method to model the advance of a internally critical salt wedge through the use of nonlinear boundary conditions gives hope that the more complex topographic response problem may also be tractable, as has been the case in the analogous barotropic problem. In this regard, a harmonic model has the advantage of suppressing the high-frequency internal waves that are generated by topography but not fully resolved, in a time-stepping model, unless the time step is very small. In our approach, the nonlocal, vertical exchange occurring as these waves propagate away from topographic features would be parameterized in a mixing calculation linked to the harmonic model. The success of an analytical model in predicting nonlinear internal wave propagation away from a sill [Farmer and Smith, 1978] suggests that such a parameterization can be devised, and it may well prove easier to apply such a parameterization as a subsidiary calculation to a two-layer harmonic model than in a finite difference or finite element, time-stepping model.

Acknowledgments. This research was supported by NSF grants OCE-8208856, OCE-8504237, and OCE-8711790. Kathy Smith typed the equations and processed the text; Lin Sylwester prepared the figures.

\section{REFERENCES}

Abraham, G., Turbulence and mixing in stratified tidal flow, in Physical Processes in Estuaries, edited by J. Dronkers and W. van Leussen, pp. 149-180, Springer-Verlag, New York, 1988. Allen, G. P., J. C. Salomon, P. Bassoullet, Y. Du Penhoat, and C. De Grandpré, Effects of tides on mixing and suspended sediment in macrotidal estuaries, Sed. Geol., 26, 69-80, 1980.

Dore, B. D., Mass transport in layered fluid systems, J. Fluid Mech., 4O(1), 113-126, 1970.

Dronkers, J. J., Tidal Computations in Rivers and Coastal Waters, pp. 263-304, North-Holland, Amsterdam, 1964.

Farmer, D., and J. D. Smith, nonlinear internal waves in a fjord, in Hydrodynamics of Estuaries and Fjords, edited by J. C. J. Nihoul, pp. 465-494, Elsevier, New York, 1978.

Gardner, G. B., A. R. M. Nowell, and J. D. Smith, Turbulent processes in estuaries, in Estuarine and Wetland Processes, edited by P. Hamilton P. and K. B. MacDonald pp. 1-34, Plenum, New York, 1980.

Gargett, A. E., Vertical eddy diffusivity in the ocean interior, $J$. Mar. Res., 42, 359-393, 1984.

Geyer, W. R., The time-dependent dynamics of a salt wedge, Ph.D. thesis, Univ. of Wash., Seattle, 1985.

Geyer, W. R., The advance of a salt wedge: observations and dynamical model, in Physical Processes in Estuaries, edited by J. Dronkers and W. van Leussen, pp. 181-195, SpringerVerlag, New York, 1988.

Geyer, W. R., and J. D. Smith, Shear instability in a highly stratified estuary, J. Phys. Oceanogr., 17(10), 1668-1679, 1987.

Haas, L. W., The effect of the neap-spring tidal cycle on the tical salinity structure of the James, York and Rappahannock Rivers, Virginia, USA, Estuarine Coastal Mar. Sci., 5, $496,1976$.

Hansen, D. V., and M. Rattray, Jr., Gravitational circulation in straits and estuaries, J. Mar. Res., 23, 104-122, 1965.

Ianniello, J. P., nonlinearly induced residual currents in tidally dominated estuaries, Ph.D. thesis, Univ. of Conn., Storrs, $1977 a$.

Ianniello, J. P., Tidally induced residual currents in estuaries of constant breadth and depth, J. Mar. Res., 35, 755-774, 1977 .

Ianniello, J. P., Tidally induced currents in estuaries of variable breadth and depth, J. Phys. Oceanogr., 9, 962-974, 1979.

Ianniello, J. P., Comments on tidally induced residual currents in estuaries: dynamics and near-bottom flow characteristics, Phys. Oceanogr., 11, 126-134, 1981.

Jay, D. A., Circulatory processes in the Columbia River Estuary, 169 pp., Columbia Riv. Est. Study Task, Astoria Oreg.,

Jay, D. A., Residual circulation in shallow, stratified estuari Ph.D. thesis, Univ. of Wash., Seattle, 1987.

Jay, D. A., Residual circulation in shallow, stratified estuaries: shear, stratification and transport processes, in Residual Currents and Long-Term Transport in Estuaries and Bays, edited by R. T. Cheng, Springer-Verlag, New York, in press, 1989.

Jay D. A., and J. D. Smith, Residual circulation in and classfication of shallow, stratified estuaries, in Physical Processes in Estuaries, edited by J. Dronkers and W. van Leussen, pp. 19-41, Springer-Verlag, New York, 1988.

Jay D. A., and J. D. Smith, Circulation, density structure and neap-spring transitions in the Columbia River Estuary, Prog. Oceanogr., in press, 1989.

Jay D. A., and J. D. Smith, Residual circulation in shallow estuaries: 2. Weakly stratified and partially mixed estuaries, this issue.

Jay D. A., B. S. Giese, and C. R. Sherwood, Columbia River Estuary: Energetics and sedimentation, Prog. Oceanogr., in press, 1989.

Lawrence, G. A., The hydraulics and mixing of two-layer flow over an obstacle, Hydraul. Eng. Lab. Rep. UCB/HEL-85/02, 122 pp., Univ. of Calif., Berkeley, 1985.

LeBlond, P. H., On tidal propagation in shallow rivers, $J$. phys. Res., 83, 4717-4721, 1978.

Neal, V. T., Physical aspects of the Columbia River and it tuary, in The Columbia River Estuary and Adjacent Ocen Waters, edited by A. T. Pruter and D. L. Alverson, pp 19-40, University of Washington Press, Seattle, 1972.

Orem, H. M., Discharge in the lower Columbia River basin, 1 65, U.S. Geol. Surv. Circ., 550, 24 pp., 1968.

Paulsen, C.G., Floods of May-June 1948 in the Columbia 
basin, Water Supply Pap., 1080, 476 pp., U.S. Geol. Surv., Reston, Va., 1949.

Sherwood, C. R., D. A. Jay, R. B. Harvey, P. Hamilton and C. D. Simenstad, Historical changes in the Columbia River Estuary, Prog. Oceanogr., in press, 1989.

Stigebrandt, A., A mechanism governing the estuarine circulation in deep, strongly stratified fjords, Estuarine Coastal Mar. Sci., 13, 197-211, 1981.

Stokes, G. G., On the theory of oscillatory waves, Trans. Cambridge Philos. Soc., 8, 441-455, 1847.

Zimmerman, J. T. F., Vorticity transfer by tidal currents over an irregular topography, J. Mar. Res., 38(4), 601-630, 1980.

Zimmerman, J. T. F., The tidal whirlpool: a review of horizontal dispersion by tidal and residual currents, Neth. J. Sea Res., 20(2/3), 133-154, 1986.

D. A. Jay and J. D. Smith, Geophysics Program, AK-50, University of Washington, Seattle, WA 98195.

(Received April 13, 1988; accepted August 29, 1988.) 